\title{
The MOLAR Project: atmospheric deposition and lake water chemistry
}

\author{
The MOLAR Water Chemistry Group ${ }^{1}$ \\ Correspondence: R. Mosello, CNR Istituto Italiano di Idrobiologia, L.go Tonolli 50, 28922 Verbania Pallanza, Italy \\ e-mail: r.mosello@iii.to.cnr.it
}

\begin{abstract}
In the framework of the Mountain Lake Research (MOLAR) project, the water chemistry of 23 lakes covering Europe from the Svalbard Islands to the South of Spain were studied. The lakes are located above or beyond the timber line in the most important mountain areas in Europe. Atmospheric deposition was sampled at 11 sites, representative of different lake districts. 24 institutions took part in the activity. The comparability of the analytical results performed in 18 laboratories was assured by strict sampling and analysis protocols and by a detailed programme of Analytical Quality Assessment/Control. The paper summarises the main morphometric and hydrological characteristics of the lakes and their watersheds and discusses the results of the atmospheric deposition and lake water chemistry. An overview on the main processes controlling the composition and ionic ratio of deposition and lake chemistry is given.
\end{abstract}

Key words: remote lakes, water chemistry, deposition chemistry

\section{INTRODUCTION}

The Mountain Lake Research (MOLAR) project considered the most remote and least disturbed freshwater ecosystems in Europe, mainly located in the Alpine and Arctic regions. Although far from local sources of pollution, these lakes are threatened by the atmospheric deposition of pollutants (acidity and toxic air pollutants) and by climate change. The remote lakes found throughout these regions are especially sensitive to these threats for a number of related reasons:

- many are sensitive to acidification because of the poor buffering capacities of soil and rocks in the watershed;

- nitrate levels are higher because their catchments have little soil and vegetation to take up nitrogen deposition from the atmosphere;

- toxic trace metals and trace organics accumulate in the food chain more easily, and some pollutants (e.g. mercury, volatile organics) accumulate preferentially in cold regions;

- climate warming in Europe is predicted to be greatest in the Arctic and Alpine regions.

Because of their sensitivity, remote mountain lakes are not only vulnerable to environmental change, but are also excellent sensors of change, and their high quality sediment records can be used to infer the speed, direction and biological impact of changing air quality and climate.

With these characteristics as its basis, the MOLAR project recognised four overall objectives, each corresponding to a major strand or work package (WP) in the work programme:
1) to measure and model the dynamic responses of remote mountain lake ecosystems to acid (sulphur plus nitrogen) deposition;

2) to quantify and model pollutant (trace metals, trace organics) fluxes and pathways in remote mountain lakes and their uptake by fish;

3) to measure and model the temporal responses of remote mountain lake ecosystems to climate variability on seasonal, inter-annual and decadal time scales;

4) to continue the development of a high quality environmental database on remote mountain lake ecosystems in Europe and to disseminate the results widely.

The MOLAR project, carried out from February 1996 to February 1999, was funded within the EU Environmental and Climate Programme with assistance from INCO. It builds on other previous EU funded projects: AL:PE 1 (Acidification of Remote Mountain Lakes: Palaeolimnology and Ecology) and AL:PE2 (Remote Mountain Lakes as Indicators of Air Pollution and Climate Change), which represented the first comprehensive study of remote mountain lakes at a European level (Wathne et al. 1995, 1997; Battarbee et al. 1997).

Several physical, chemical and biological aspects of the MOLAR lakes were considered during the execution of the project; we refer to Patrick et al. (1998) for a general presentation. A total of 24 Institutions were involved in the project; table 1 lists those contributing to the studies on atmospheric deposition and lake water chemistry, and the personnel responsible for the chemistry activities.

This paper aims to present the results obtained on the chemical fluxes of acid compounds (sulphur and ni-

\footnotetext{
${ }^{1}$ See table 1 for the Authors' list.
} 
Tab. 1. Institutions participating in the MOLAR project, acronyms of the Institutes and studied lakes. Lake acronyms refer to table 2.

\begin{tabular}{|c|c|c|c|}
\hline Institute involved & $\begin{array}{l}\text { Institute } \\
\text { (acronym) }\end{array}$ & $\begin{array}{c}\text { Lake } \\
\text { (acronym) }\end{array}$ & Persons in charge \\
\hline Norwegian Institute for Water Research, Oslo (N) & NIVA & $\begin{array}{l}\text { Nea, Stav, } \\
\text { Arr, Lim }\end{array}$ & B. Wathne, L. Lien, L.B. Skancke \\
\hline University College London, Environ. Change Research Centre (UK) & $\mathrm{UCL}$ & Loc & N. Rose \\
\hline Freshwater Fisheries Laboratory, Pitlochry, Scotland (UK) & FFL & Loc & R. Harriman \\
\hline C.N.R. Istituto Italiano di Idrobiologia, Pallanza (I) & CNR & $\mathrm{PaS}, \mathrm{PaI}$ & $\begin{array}{l}\text { R. Mosello, A. Boggero, A. Marchetto, A. Lami, } \\
\text { G.A. Tartari, M. Rogora }\end{array}$ \\
\hline Laboratorio Biologico Provinciale, Laives (I) & LBP & Lat & D. Tait, B. Thaler \\
\hline Univ. Bordeaux, Lab. Neurobiol. Physiologie Comparées, Arcachon (F) & CNRS & Aub & J.C. Massabuau \\
\hline University of Innsbruck, Inst. of Zoology and Limnology (A) & UIBK & SoS, Gks & $\begin{array}{l}\text { R. Psenner, H. Thies, S. Sommaruga-Wögrath, K.A. } \\
\text { Koinig }\end{array}$ \\
\hline University of Innsbruck, Inst. of Meteorology and Geophysics (A) & UIMG & Gks & U. Nickus \\
\hline Universitat de Barcelona, Dept. Ecologia (E) & UB-DE & Red & J. Catalan, L. Camarero, M. Ventura \\
\hline Universidad de Granada, Instituto del Agua (E) & UGR & Cal & L. Cruz Pizarro, P. Carillo, M. Villar, J.M. Medina \\
\hline Charles University, Dept. Hydrobiology, Prague (CZ) & FSCU & Star, Niz & E. Stuchlík, J. Fott, O. Strunecký \\
\hline Hydrobiological Institute Czech Rep, Česke Budějovice (CZ) & ASCR & Star, Niz & J. Kopáček, V. Straškrabová \\
\hline Inst. of Zoology, Slovak Academy of Sciences, Bratislava (SK) & IZ-SAS & Star, Niz & F. Šporka \\
\hline Faculty of Ecol. and Env. Sciences, Tech. University in Zvolen (SK) & FEES & Star, Niz & P. Bitušík \\
\hline Inst. Freshwater Biology, Polish Academy of Sciences, Kracow (PL) & IFB-PAS & Dlu & J. Galas \\
\hline Universidad Autonoma Madrid, Dept. Ecologia (E) & UAM & Cim & I. Granados \\
\hline Inst. Ecological Problems, Kola Science Centre, Apatity (SU) & INEP & Chu & T.I. Moiseenko, Kudryavtieva Lubov \\
\hline National Institute of Biology, Ljubljana (SLO) & NIB & Led & A. Brancelj, G. Muri, A. Gaberscik \\
\hline S.P.A.A. Laboratorio Studi Ambientali, Canton Ticino $(\mathrm{CH})$ & LSA & Lag & A. Barbieri \\
\hline University of Helsinki, Dept. of Geography (FIN) & UHEL & Saa & A. Korhola, S. Sorvari, M. Rautio, J. Virkanen \\
\hline Swiss Federal Institute for Envir. Sci. and Technol., Dübendorf $(\mathrm{CH})$ & EAWAG & Hag & A.F. Lotter \\
\hline Limnological Research Centre, EAWAG, Kastanienbaum (CH) & EAWAG & Hag & B. Müller \\
\hline University of Bern, Lab. Environmental and Radiochemistry $(\mathrm{CH})$ & UBERN & Jor & B. Steiner, U. Krähenbühl \\
\hline University of Zürich, Inst. Plant Biology $(\mathrm{CH})$ & Uzuerich & Jor & M. Gabathuler, K. Hanselmann \\
\hline
\end{tabular}

trogen) from the atmosphere and the lake water chemistry. It will consider the general features of the lakes and their watersheds, and the characteristics of the sites where atmospheric deposition was sampled. It will also describe sampling methods and treatment of samples and the inter-laboratory studies performed to assure the comparability of chemical results. Separate papers have considered the chemistry and atmospheric deposition of each site; water chemistry is also considered in a different part of the project dealing with modelling. For this reason we consider it useful to collect all the available information concerning methods and site characteristics in one paper, which can also be used as a reference for the other Work Pakages (WPs) making up the MOLAR project.

\section{METHODS}

\subsection{Sampled lakes}

All the studied lakes (Fig. 1, Tab. 2) are above or beyond the timber line and are covered with ice for a period during winter. The lakes are located in a range of longitude from $5^{\circ} 18^{\prime} \mathrm{W}$ (Laguna Cimera) to $32^{\circ} 30^{\prime} \mathrm{E}$ (Chuna), latitudes range from $37^{\circ} 03^{\prime}$ (La Caldera) to $79^{\circ} 40^{\prime}$ (Arresjøen); the altitudes of the latter two lakes are also at the extremes, being at 3050 and $20 \mathrm{~m}$ a.s.l. respectively. Lake surfaces range from 0.7 ha (Starolesnianske pleso) to 70 ha (Saanajärvi); 15 of the 23 lakes have a surface area of less than 10 ha. The range of maximum and mean depths is 4.1-73 $\mathrm{m}$ and 1.6-32 $\mathrm{m}$ respectively. The amount of precipitation in the watersheds ranges from $350 \mathrm{~mm} \mathrm{y}^{-1}$ (Limgambergtjern) to over $2000 \mathrm{~mm} \mathrm{y}^{-1}$ for lakes in the Alps and Pyrenees (Laghetto Inferiore, Jezero v Ledvici, Étang d'Aubé) (Tab. 3). The hydrology is strongly influenced by the long period of ice cover during the winter, as most of the lakes are ice-free only from June to October, and the period of maximum hydrological activity is during the snowmelt, from April to July. These seasonal variations are of great importance in determining the hydrology, so that the mean annual values of lake water retention time and annual runoff (Tab. 3 ) should be considered only as indicative.

Most of the watersheds of the MOLAR lakes (Tab. 4) are formed of gneiss (7 lakes) or granite (7 lakes), while three of them are formed mainly of calcareous rocks (Jezero v Ledvici, Saanajärvi, Hagelsee). Soils are thin and constitute only a fraction of the whole watershed, as bare rocks, moraine and debris are an important component of the drainage basin. In most cases the vegetation consists of alpine meadows, with a few exceptions where sparse trees are present (Tab. 4).

\subsection{Sampling of atmospheric deposition (rain, snow)}

The flux of ions and nutrients from the atmosphere to the watershed/lake surface was evaluated by sampling and analysis of direct deposition and snowpack. All aspects of sampling, field and laboratory measurements were described in detail in a manual prepared by the various specialists at the beginning of the project (Wathne \& Hansen 1997). In particular, atmospheric 
Lakes with atmospheric deposition sampling station

Lakes without atmospheric deposition sampling station

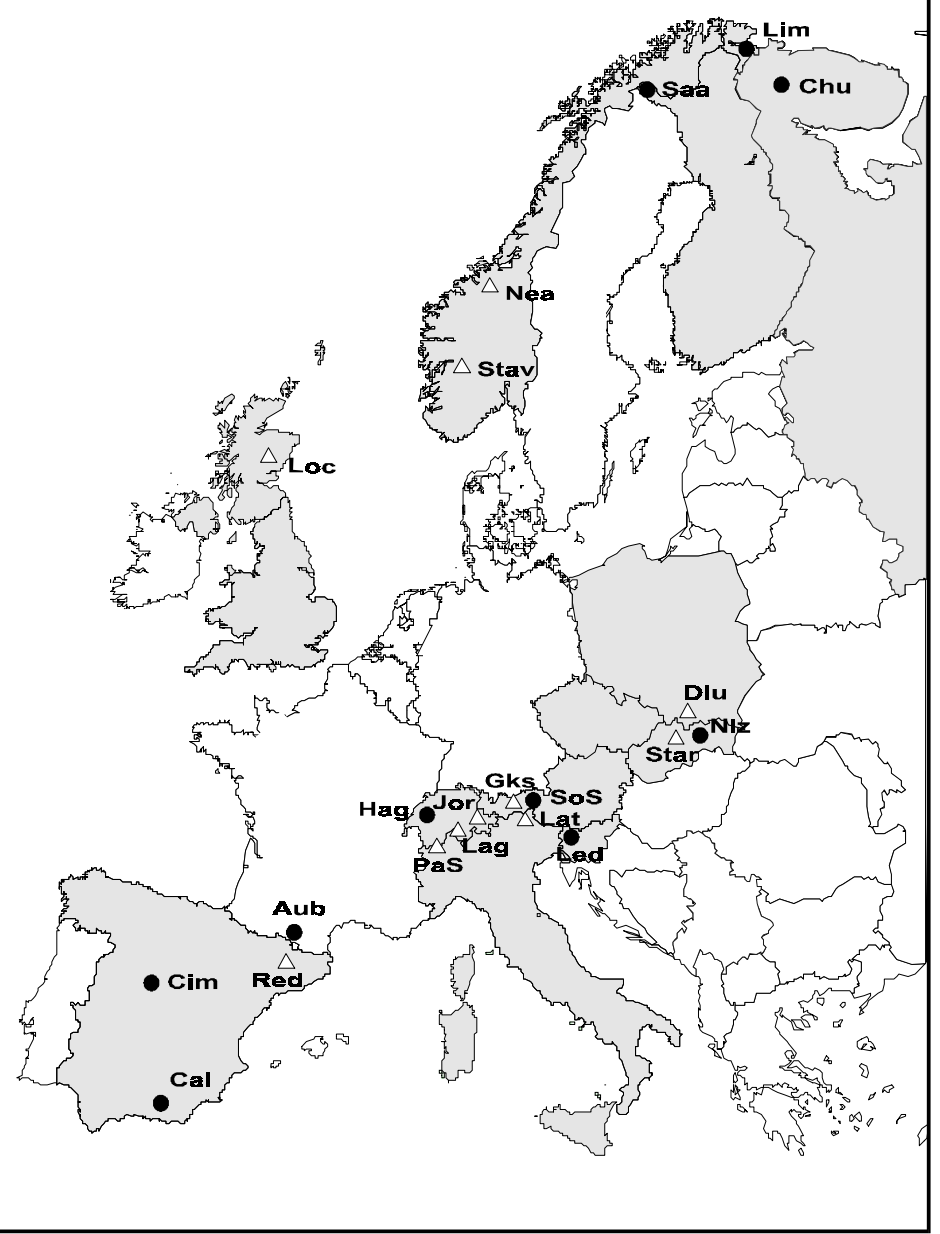

Fig. 1. Location of the MOLAR

lakes (acronyms refer to table 2) and atmospheric deposition sampling stations. deposition, including sampling and analysis of direct deposition and snow pack, was dealt with by Mosello et al. (1997a). The primary goal of these measurements is to provide a basis for evaluation of the total deposition of atmospheric pollutants, as this variable plays a fundamental role for most of the modelling, including comparisons with critical loads. The characteristics of the sampling stations, their distance from the lake and eventual local disturbances are reported in table 5. Each site was chosen to be representative of the atmospheric deposition on the lake/watershed surface, and not greatly affected by local sources of pollution that might lead to misinterpretation of the chemical results.
Wet-only sampling was chosen for the sites where electric power is available; alternatively, bulk sampling was used (two sites). Sampling frequency varied according to the accessibility of the sites, as indicated in table 6. Heavy snow events were sampled using a cylindrical bulk collector, made of polyethylene and 1.5 $\mathrm{m}$ high, raised about $0.5 \mathrm{~m}$ off the ground.

A study on the differences between wet-only and bulk deposition chemistry was carried out in the framework of the MOLAR project at Lake Redó, in the Pyrenees. Precipitation samples were collected using a bulk deposition collector, consisting of a polypropylene funnel with a diameter of $28 \mathrm{~cm}$ and a collection tank 
Tab. 2. Morphometric characteristics of the MOLAR lakes. See table 1 for the explanation of the acronyms.

\begin{tabular}{|c|c|c|c|c|c|c|c|c|c|c|c|c|}
\hline Lake & Acron. & Country & Institute & $\begin{array}{l}\text { Analytical } \\
\text { laboratory }\end{array}$ & Latitude & Longitude & $\begin{array}{l}\text { Lake alt. } \\
\text { (m a.s.l.) }\end{array}$ & $\begin{array}{c}\text { Cat. area } \\
\left(\mathrm{km}^{2}\right)\end{array}$ & $\begin{array}{l}\text { Lake } \\
\text { (ha) }\end{array}$ & $\begin{array}{l}\text { Max depth } \\
\text { (m) }\end{array}$ & $\begin{array}{l}\text { Mean depth } \\
\text { (m) }\end{array}$ & $\begin{array}{l}\text { h Volume } \\
\left(\mathrm{m}^{3} 10^{6}\right)\end{array}$ \\
\hline Øvre Neådalsvatn & Nea & $\mathrm{N}$ & NIVA & NIVA & $62^{\circ} 46^{\prime} 30^{\prime \prime}$ & $09^{\circ} 00^{\prime} \mathrm{E}$ & 728 & 16.00 & 50.0 & 18.0 & 3.9 & 1.95 \\
\hline Stavsvatn & Stav & $\mathrm{N}$ & NIVA & NIVA & $59^{\circ} 38^{\prime}$ & $08^{\circ} 07^{\prime} \mathrm{E}$ & 1053 & 2.43 & 40.0 & 17.0 & 6.2 & 2.50 \\
\hline Lochnagar & Loc & UK & UCL & FFL & $56^{\circ} 57^{\prime} 29^{\prime \prime}$ & $03^{\circ} 13^{\prime} 05^{\prime \prime} \mathrm{W}$ & 785 & 1.02 & 9.8 & 24.0 & 8.4 & 0.82 \\
\hline Paione Superiore & $\mathrm{PaS}$ & I & $\mathrm{CNR}$ & CNR & $46^{\circ} 10^{\prime} 26^{\prime \prime}$ & $08^{\circ} 11^{\prime} 27^{\prime \prime} \mathrm{E}$ & 2269 & 0.55 & 1.4 & 11.5 & 5.1 & 0.07 \\
\hline Paione Inferiore & $\mathrm{PaI}$ & I & CNR & CNR & $46^{\circ} 10^{\prime} 01^{\prime \prime} \mathrm{N}$ & $08^{\circ} 11^{\prime} 23^{\prime \prime} \mathrm{E}$ & 2002 & 1.14 & 1.4 & 13.5 & 7.4 & 0.10 \\
\hline Lago di Latte & Lat & I & LBP & LBP & $46^{\circ} 43^{\prime} 32^{\prime \prime} \mathrm{N}$ & $11^{\circ} 04^{\prime} 21^{\prime \prime} \mathrm{E}$ & 2540 & 0.65 & 2.3 & 12.3 & 5.3 & 0.13 \\
\hline Étang d'Aubé & Aub & $\mathrm{F}$ & CNRS & CNRS & $42^{\circ} 44^{\prime} 44^{\prime \prime} \mathrm{N}$ & $01^{\circ} 20^{\prime} \mathrm{E}$ & 2091 & 0.77 & 8.6 & 45.0 & - & - \\
\hline Arresjøen & Arr & $\mathrm{N}$ & NIVA & NIVA & $79^{\circ} 40^{\prime} \mathrm{N}$ & $10^{\circ} 48^{\prime} \mathrm{E}$ & 20 & 3.00 & 35.0 & 32.0 & 10.9 & 3.80 \\
\hline Schwarzsee ob Sölden & SoS & A & UIBK & UIBK & $47^{\circ} 51^{\prime} 56^{\prime \prime} \mathrm{N}$ & $10^{\circ} 56^{\prime} 45^{\prime \prime} \mathrm{E}$ & 2799 & 0.14 & 3.4 & 18.0 & 10.0 & 0.34 \\
\hline Gossenköllesee & Gks & A & UIBK & IMG / IZL & $47^{\circ} 13^{\prime} 49^{\prime \prime} \mathrm{N}$ & $11^{\circ} 00^{\prime} 51^{\prime \prime} \mathrm{E}$ & 2417 & 0.01 & 1.7 & 9.9 & 4.7 & 0.08 \\
\hline Redó & Red & $\mathrm{E}$ & UB & $\mathrm{DE}$ & $42^{\circ} 38^{\prime} 34^{\prime \prime} \mathrm{N}$ & $00^{\circ} 46^{\prime} 13^{\prime \prime} \mathrm{E}$ & 2240 & 1.55 & 24.0 & 73.0 & 32.0 & 7.75 \\
\hline La Caldera & Cal & $\mathrm{E}$ & UGR-ES & UGR-ES & $37^{\circ} 03^{\prime} \mathrm{N}$ & $03^{\circ} 20^{\prime} \mathrm{W}$ & 3050 & 0.18 & 2.3 & 11.3 & 4.6 & 0.11 \\
\hline Starolesnianske pl. & Star & SK & FSCU & $\begin{array}{c}\text { FSCU/HBI } \\
\text {-ASCR }\end{array}$ & $49^{\circ} 10^{\prime} \mathrm{N}$ & $20^{\circ} 10^{\prime} \mathrm{E}$ & 2000 & 0.03 & 0.7 & 4.1 & 1.6 & 0.01 \\
\hline Nižné Terianske pl. & $\mathrm{Niz}$ & SK & IZ-SAS & $\begin{array}{c}\text { FSCU/HBI } \\
\text {-ASCR }\end{array}$ & $49^{\circ} 10^{\prime} \mathrm{N}$ & $20^{\circ} 00^{\prime} \mathrm{E}$ & 1941 & 1.10 & 4.8 & 44.4 & 18.4 & 0.89 \\
\hline Długi Staw & Dlu & PL & IFB-PAS & IFB-PAS & $49^{\circ} 13^{\prime} 36^{\prime \prime} \mathrm{N}$ & $20^{\circ} 00^{\prime} 39^{\prime \prime} \mathrm{E}$ & 1783 & 0.66 & 1.6 & 10.6 & 5.1 & 0.08 \\
\hline Laguna Cimera & Cim & $\mathrm{E}$ & UAM & UAM & $40^{\circ} 15^{\prime} 50^{\prime \prime} \mathrm{N}$ & $05^{\circ} 18^{\prime} 15^{\prime \prime} \mathrm{W}$ & 2140 & 0.85 & 4.5 & 9.4 & 4.8 & 0.22 \\
\hline z. Chuna & Chu & SU & INEP & INEP & $67^{\circ} 55^{\prime} \mathrm{N}$ & $32^{\circ} 30^{\prime} \mathrm{E}$ & 420 & 2.05 & 12.5 & 18.0 & 10.0 & 1.25 \\
\hline Jezero v Ledvici & Led & SLO & NIB & NIB & $46^{\circ} 20^{\prime} 30^{\prime \prime} \mathrm{N}$ & $13^{\circ} 47^{\prime} 20 \mathrm{E}$ & 1830 & - & 2.4 & 15.0 & 5.7 & 0.14 \\
\hline Limgambergtjern & Lim & $\mathrm{N}$ & NIVA & NIVA & $69^{\circ} 41^{\prime} \mathrm{N}$ & $30^{\circ} 21^{\prime} \mathrm{E}$ & 172 & - & 14.0 & 14.0 & 4.1 & 0.57 \\
\hline Saanajärvi & Saa & SF & UHEL & UHEL & $69^{\circ} 05^{\prime} \mathrm{N}$ & $21^{\circ} 27^{\prime} \mathrm{E}$ & 679 & 4.60 & 70.0 & 24.0 & - & - \\
\hline Hagelsee & Hag & $\mathrm{CH}$ & EAWAG & EAWAG & $46^{\circ} 40^{\prime} \mathrm{N}$ & $08^{\circ} 02^{\prime} \mathrm{E}$ & 2339 & 0.36 & 3.0 & 18.5 & 8.3 & 0.19 \\
\hline Jörisee & Jor & $\mathrm{CH}$ & Uzuerich & UBERN & $46^{\circ} 46^{\prime} 40^{\prime \prime} \mathrm{N}$ & $09^{\circ} 57^{\prime} 20^{\prime \prime} \mathrm{E}$ & 2490 & 1.10 & 57.8 & 21.8 & 10.5 & 0.60 \\
\hline Laghetto Inferiore & Lag & $\mathrm{CH}$ & LSA & LSA & $46^{\circ} 28^{\prime} 37^{\prime \prime} \mathrm{N}$ & $08^{\circ} 35^{\prime} 38^{\prime \prime} \mathrm{E}$ & 2074 & 1.78 & 4.8 & 33.0 & 10.6 & 0.65 \\
\hline
\end{tabular}

Tab. 3. Hydrology and atmospheric loads of $\mathrm{S}$ and $\mathrm{N}$ for the MOLAR lakes. * (July-October) approximating that $70 \%$ of the deposition occurred as snow (no evaporation correction)

\begin{tabular}{|c|c|c|c|c|c|c|c|}
\hline Lake & Country & $\begin{array}{l}\text { Precipitation } \\
\left(\mathrm{mm} \mathrm{y}^{-1}\right)\end{array}$ & $\begin{array}{l}\text { Retention time } \\
\text { (d) }\end{array}$ & $\begin{array}{l}\text { Annual runoff } \\
\left(1 \mathrm{~s}^{-1} \mathrm{~km}^{-2}\right)\end{array}$ & Ice free period & $\begin{array}{c}\text { Deposition (S) } \\
\left(\mathrm{g} \mathrm{S} \mathrm{m}^{-2} \mathrm{y}^{-1}\right)\end{array}$ & $\begin{array}{c}\text { Deposition (N) } \\
\left(\mathrm{g} \mathrm{N} \mathrm{m}^{-2} \mathrm{y}^{-1}\right)\end{array}$ \\
\hline$\emptyset_{\text {vre Neådalsvatn }}$ & $\mathrm{N}$ & 1500 & 24 & 60 & June-October & 0.24 & 0.35 \\
\hline Stavsvatn & $\mathrm{N}$ & 1000 & 380 & 31 & June-Sept./Oct. & 0.55 & 0.50 \\
\hline Lochnagar & UK & 1034 & 315 & 30 & ca. 8 months & 0.47 & - \\
\hline Paione Superiore & I & 1400 & 33 & 44 & August-October & $0.4-0.6$ & - \\
\hline Paione Inferiore & I & 1450 & 23 & 46 & July-October & $0.4-0.6$ & - \\
\hline Lago di Latte & I & 1000 & 71 & 31 & July-October & 0.53 & - \\
\hline Étang d'Aubé & $\mathrm{F}$ & $2500-3000$ & - & $>1000 \mathrm{~mm}$ & June-October & - & - \\
\hline Arresjøen & $\mathrm{N}$ & 500 & 1095 & 13 & July-September & 0.17 & 0.13 \\
\hline Schwarzsee ob Sölden & A & - & not available & not available & July/August-October & - & - \\
\hline Gossenköllesee & A & 1200 & $\begin{array}{l}\text { tracer exper. still } \\
\text { running }\end{array}$ & - & Jun/Jul - Oct/Nov & - & - \\
\hline Redó & $\mathrm{E}$ & $1300-1500$ & 1217-1404 & $41-48$ & June-December & 1.0 & 0.44 \\
\hline La Caldera & $\mathrm{E}$ & 710 & 390 & 18 & June-October & - & - \\
\hline Starolesnianske & SK & 1145 & 134 & 33 & June-September & 1.00 & 0.7 \\
\hline Nižné Terianske & SK & 1145 & 293 & 33 & Juli-October & 1.00 & 0.7 \\
\hline Długi Staw & PL & 1800 & 34 & 42 & May-July/Sept-Nov & 2.60 & 1.26 \\
\hline Laguna Cimera & $\mathrm{E}$ & 1700 & 62 & 46 & June-Oct/Nov & - & - \\
\hline z. Chuna & SU & 1000 & - & 18 & June-October & $0.6-1.5$ & - \\
\hline Jezero v Ledvici & SLO & $2500-2800$ & - & - & May-Nov & - & - \\
\hline Limgambergtjern & $\mathrm{N}$ & 350 & 330 & 17.00 & June-October & 0.7 & 0.25 \\
\hline Saanajärvi & SF & - & - & - & July-October & - & - \\
\hline Hagelsee & $\mathrm{CH}$ & 1700 & - & - & August-October & - & - \\
\hline Jörisee & $\mathrm{CH}$ & $1100-1400$ & 120 & 50 & June-October & $0.28 *$ & $0.52 *$ \\
\hline Laghetto Inferiore & $\mathrm{CH}$ & 2300 & 55 & 60 & July-October & 0.72 & 0.9 \\
\hline
\end{tabular}

with a capacity of 51 . A $200 \mu \mathrm{m}$ nylon mesh was placed between the funnel and the tank to filter out coarse particles. The sampler was emptied daily, the total collected volume measured and a subsample taken for chemical analysis. In order to assess the amount of dry deposition collected by the sampler, on days when no precipitation water was collected, funnel and bottle were carefully rinsed with a known amount of de-ionised water and a sample of this rinsing water taken for chemical analysis. Sampling was performed from 5 July 1997 until 11 January 1998.

Snowpack was sampled at several sites generally not easily accessible during winter. It was especially important to sample the total snow pack before the first 
Tab. 4. Characteristics of the watersheds and notes on fish presence of the MOLAR lakes.

\begin{tabular}{|c|c|c|c|c|}
\hline Lake & Cat. geology & Cat. soil & Cat. vegetation & Fish presence \\
\hline Øvre Neådalsvatn & Gneiss & Alpine soil & $\begin{array}{l}\text { Alpine heath, pasture, bare } \\
\text { rock }\end{array}$ & Brown trout, reproduction \\
\hline Stavsvatn & Granite & Alpine soils and peat & $\begin{array}{l}\text { Rocks, pasture, alpine heath, } \\
\text { some birch trees }\end{array}$ & Brown trout, no reproduction \\
\hline Lochnagar & Granite & Peat and alpine soil & $\begin{array}{c}\text { Dwarf shrub (alpine) heath, } \\
\text { bare rock }\end{array}$ & $\begin{array}{c}\text { Brown trout, reproduction in } \\
\text { outflow }\end{array}$ \\
\hline Paione Superiore & Gneiss & Alpine soil & $\begin{array}{l}\text { Rocks and debris, some hay } \\
\text { meadows }\end{array}$ & No fish \\
\hline Paione Inferiore & Gneiss & Alpine soil & $\begin{array}{l}\text { Rocks and debris, some hay } \\
\text { meadows }\end{array}$ & Rainbow trout \\
\hline Lago di Latte & Gneiss & Alpine soil & $\begin{array}{l}\text { Bare rocks, alpine pasture, } \\
\text { permanent ice }\end{array}$ & Arctic char (reproduction) \\
\hline Étang d'Aubé & Granite & - & Rocks, debris, some pasture & $\begin{array}{l}\text { Brown trout, no reproduction, lake } \\
\text { char, rep. very dace, no rep. }\end{array}$ \\
\hline Arresjøen & Migmatite & Permanent frozen & Rocks, debris & Arctic char, (landlocked with rep.) \\
\hline Schwarzsee ob Sölden & Gneiss, granite, diorit & Bare rocks, thin alpine soil & Rocks, alpine mats & Arctic char (reduced reproduction) \\
\hline Gossenköllesee & Gneiss, granite, amphibolites & Alpine and podsolic rankers & Alpine meadows and bare rock & Brown trout \\
\hline Redó & $\begin{array}{c}\text { Granodiorite, rocks bearing } \\
\text { calcite }\end{array}$ & Bare rocks, ranker soil & $\begin{array}{c}\text { Festuca eskia fields, few } \\
\text { mosses }\end{array}$ & Brown trout \\
\hline La Caldera & $\begin{array}{l}\text { Micaschists, brechoid, } \\
\text { marbles, amphibolites }\end{array}$ & $\begin{array}{l}\text { Distryc cambisols, distryc } \\
\text { regosols }\end{array}$ & Rocks, debris & No fish \\
\hline Starolesnianske pl. & Granite & Primitive soil & $\begin{array}{l}\text { Alpine meadows and bare } \\
\text { rocks }(3: 1)\end{array}$ & No fish \\
\hline Nižné Terianske pl. & Granite & Primitive soil & $\begin{array}{c}\text { Alpine meadows and bare } \\
\text { rocks }(1: 1)\end{array}$ & No fish \\
\hline Długi Staw & $\begin{array}{c}\text { Granitoids covered by } \\
\text { moraine }\end{array}$ & Primitive soil-podsolic soil & $\begin{array}{c}\text { Dwarf pine, alpine meadows, } \\
\text { rocks }\end{array}$ & $\begin{array}{c}\text { No fish (brook trout stocked in } \\
1960 \text { ) }\end{array}$ \\
\hline Laguna Cimera & Granite & $\begin{array}{l}\text { Stone, hydromorphic and } \\
\text { psychroxerophilous soils }\end{array}$ & $\begin{array}{c}\text { Rocks and debris with small } \\
\text { alpine meadows }\end{array}$ & Brook trout (Salvelinus fontinalis) \\
\hline z. Chuna & Bazalts & Tundra & Tundra & Brown trout \\
\hline Jezero v Ledvici & Jurassic limestone & Alpine soil & $\begin{array}{c}\text { Alpine meadows, dwarf pine, } \\
\text { European larch }\end{array}$ & No fish \\
\hline Limgambergtjern & - & - & $1-$ & - \\
\hline Saanajärvi & $\begin{array}{c}\text { Paleozoic Caledonian schist } \\
\text { and gneiss, dolomitic } \\
\text { limestone and sedimentary } \\
\text { rocks }\end{array}$ & - & $\begin{array}{l}\text { Subalpine vegetation, bare } \\
\text { rock, boulder fields }\end{array}$ & $\begin{array}{l}\text { Arctic char (introduced), lake trout } \\
\text { (Salmo trutta lacustris) }\end{array}$ \\
\hline Hagelsee & Jurassic limestone & Alpine soil & Alpine meadows and bare rock & No fish \\
\hline Jörisee & Granite & Podsol $10 \%$ & Alpine grass tundra & $\begin{array}{c}\text { Brown trout and Salvelinus } \\
\text { namaicush }\end{array}$ \\
\hline Laghetto Inferiore & $\begin{array}{l}\text { Gneiss alkalipheldsphat, } \\
\text { anphibolite, presence of } \\
\text { carbonate }\end{array}$ & Podsol 7\% & $\begin{array}{l}\text { Rocks and debris, some hay } \\
\text { meadows and sparse shrubs }\end{array}$ & $\begin{array}{l}\text { Brown trout by artificial } \\
\text { immission }\end{array}$ \\
\hline
\end{tabular}

snowmelt in order to evaluate the amount of deposited substance load between the first snow fall (October to December) and the end of the accumulation period (February to April). The main criterion for the selection of the site was the representativity of the catchment area as regards to snow accumulation. In addition, the site should not be influenced by avalanches or frequent snowdrift, causing either additional snow accumulation or ablation. To take the snow samples, a pit $1 \times 2 \mathrm{~m}$ wide was dug and the samples taken along a vertical profile. Density, stratigraphy and temperature of the snow cover was determined in some of the sites (e.g., Gossenköllesee). The results of these measurements are presented elsewhere (Nickus et al. 1998). All material expected to come into contact with the sampled snow was carefully cleaned in the lab before use (diluted $\mathrm{HCl}$, soaked and rinsed with de-ionised water). During sam- pling fibrous cloths were avoided, and mouth masks and clean rubber gloves were used. Samples were keep frozen until analysed (e.g., Nickus et al. 1997).

\subsection{Sampling of surface water}

Water chemistry data used in this paper refer to the results obtained in WP 1, relative to surface water. Details on the sampling methods are given by Mosello et al. (1997b). In most of the lakes, in the framework of WP 2 and 3, other depths were also sampled; how far the surface concentrations are representative of the whole water column will be discussed in the text. Sampling frequencies varied according to the accessibility of the lake, from 1-2 to 12 or more samplings per year. Details for each lake are given in table 9. In this paper, mean concentrations obtained in the period June 1996July 1998 will be mainly used; however, seasonal and 
Tab. 5. Characteristics of the atmospheric deposition sampling sites.

\begin{tabular}{|c|c|c|c|c|c|c|c|c|c|c|c|c|}
\hline $\begin{array}{l}\text { Atm. deposition } \\
\text { sampling site }\end{array}$ & Country & $\begin{array}{l}\text { In charge } \\
\text { person }\end{array}$ & $\begin{array}{l}\text { In charge } \\
\text { Institute }\end{array}$ & $\begin{array}{c}\text { Lake } \\
\text { acronym }\end{array}$ & Latitude & Longitude & $\begin{array}{l}\text { Altitude } \\
\text { (m a.s.l.) }\end{array}$ & $\begin{array}{l}\text { Distance } \\
\text { from lake }\end{array}$ & $\begin{array}{l}\text { Accessibility } \\
\text { months }\end{array}$ & $\begin{array}{l}\text { Period of } \\
\text { measurement }\end{array}$ & $\begin{array}{l}\text { Number } \\
\text { of samples }\end{array}$ & Local disturbs \\
\hline Kårvatn & $\mathrm{N}$ & Berg $\mathrm{T}$. & NILU & Nea & $62^{\circ} 47^{\prime} \mathrm{N}$ & $08^{\circ} 53^{\prime} \mathrm{E}$ & 210 & 6 & 11 & $\begin{array}{l}16 / 01 / 96- \\
30 / 04 / 98\end{array}$ & 433 & - \\
\hline Møsvatn & $\mathrm{N}$ & Berg T. & NILU & Stav & $59^{\circ} 38^{\prime} \mathrm{N}$ & $08^{\circ} 07^{\prime} \mathrm{E}$ & 940 & 30 & 12 & $\begin{array}{c}01 / 07 / 96- \\
01 / 05 / 98\end{array}$ & 102 & - \\
\hline Lochnagar & UK & Rose N. & UCL & Loc & $56^{\circ} 57^{\prime} \mathrm{N}$ & $03^{\circ} 13^{\prime} \mathrm{W}$ & 785 & 0 & $11-12$ & $\begin{array}{l}29 / 08 / 96- \\
07 / 10 / 98\end{array}$ & 66 & Occasional tourists \\
\hline Starolesnianske pl. & SK & Stuchlik E. & FSCU & Star & $49^{\circ} 18^{\prime} \mathrm{N}$ & $20^{\circ} 23^{\prime} \mathrm{E}$ & 2000 & 0 & 12 & $\begin{array}{l}15 / 08 / 96- \\
22 / 06 / 98\end{array}$ & 52 & Chalet with heating \\
\hline Skalnaté pl. & SK & Stuchlik E. & FSCU & Star, Niz & $49^{\circ} 18^{\prime} \mathrm{N}$ & $20^{\circ} 23^{\prime} \mathrm{E}$ & 1778 & $5-12$ & 12 & $\begin{array}{c}08 / 11 / 96- \\
16 / 7 / 98\end{array}$ & 74 & $\begin{array}{l}\text { Astronomical observatory } \\
\text { (electric power) }\end{array}$ \\
\hline Hala Gasienicowa & PL & Galas J. & IFP-PAS & Dlu & $49^{\circ} 15^{\prime} \mathrm{N}$ & $20^{\circ} 00^{\prime} \mathrm{E}$ & 1520 & 2 & $5-6$ & $\begin{array}{c}01 / 05 / 96- \\
01 / 08 / 98\end{array}$ & 112 & Tourists \\
\hline Gossenköllesee & A & Nickus U. & UIMG & SoS, Gks & $46^{\circ} 14^{\prime} \mathrm{N}$ & $10^{\circ} 01^{\prime} \mathrm{E}$ & 2417 & 15 & 12 & $\begin{array}{l}01 / 10 / 96- \\
30 / 09 / 98\end{array}$ & 76 & Tourists \\
\hline Texel & I & Tait D. & LBP & Lat & $46^{\circ} 44^{\prime} \mathrm{N}$ & $11^{\circ} 05^{\prime} \mathrm{E}$ & 2390 & 0.7 & 12 & $\begin{array}{c}02 / 10 / 95- \\
09 / 12 / 97\end{array}$ & 15 & - \\
\hline Renon/Ritten & I & Tait D. & LBP & Lat & $46^{\circ} 35^{\prime} \mathrm{N}$ & $11^{\circ} 26^{\prime} \mathrm{E}$ & 1780 & 30 & 12 & $\begin{array}{l}08 / 01 / 96- \\
29 / 12 / 97\end{array}$ & $\begin{array}{l}\text { W } 80 \text { B } \\
78\end{array}$ & - \\
\hline Jörisee & $\mathrm{CH}$ & Steiner B. & UBERN & Jor & $46^{\circ} 47^{\prime} \mathrm{N}$ & $09^{\circ} 57^{\prime} \mathrm{E}$ & 2500 & 0 & $4 / 5$ & $\begin{array}{l}12 / 07 / 96- \\
17 / 10 / 97\end{array}$ & 67 & Tourists \\
\hline Robiei & $\mathrm{CH}$ & Barbieri A. & LSA & Lag & $46^{\circ} 27^{\prime} \mathrm{N}$ & $08^{\circ} 31^{\prime} \mathrm{E}$ & 1890 & 7 & 12 & $\begin{array}{l}26 / 06 / 96- \\
27 / 07 / 98\end{array}$ & 52 & Cable way (electric power) \\
\hline Graniga & I & Mosello R. & CNR & $\mathrm{PaS}, \mathrm{PaI}$ & $46^{\circ} 07^{\prime} \mathrm{N}$ & $08^{\circ} 11^{\prime} \mathrm{E}$ & 1080 & 5 & 12 & $\begin{array}{l}01 / 01 / 96- \\
26 / 10 / 98\end{array}$ & 103 & Paved road \\
\hline Redó & $\mathrm{E}$ & Camarero L. & UB-DE & Red & $42^{\circ} 39^{\prime} \mathrm{N}$ & $00^{\circ} 46^{\prime} \mathrm{E}$ & 2240 & 0 & 12 & $\begin{array}{l}07 / 06 / 97- \\
05 / 07 / 98\end{array}$ & 13 & - \\
\hline CRAM & $\mathrm{E}$ & Camarero L. & UB-DE & Red & $42^{\circ} 39^{\prime} \mathrm{N}$ & $00^{\circ} 46^{\prime} \mathrm{E}$ & 1600 & 1 & 12 & $\begin{array}{l}08 / 04 / 97- \\
05 / 07 / 98\end{array}$ & 186 & $\begin{array}{l}\text { Paved road, chalet with } \\
\text { heating }\end{array}$ \\
\hline
\end{tabular}

Tab. 6. Characteristics of the area surrounding the MOLAR atmospheric deposition sampling sites.

\begin{tabular}{|c|c|c|c|c|c|c|}
\hline $\begin{array}{l}\text { Atm. deposition } \\
\text { sampling site }\end{array}$ & Geo-lithology & Vegetal cover & Sampler & Frequency & $\begin{array}{c}\text { Snowpack } \\
\text { measurement }\end{array}$ & $\begin{array}{l}\text { Precipitation amount } \\
\text { measurement }\end{array}$ \\
\hline Kårvatn & Gneiss, quartzite & Pine, pasture & Bulk & Daily & Yes & $\begin{array}{c}\text { meteo station/weight of } \\
\text { samples }\end{array}$ \\
\hline Møsvatn & $\begin{array}{l}\text { Metarhyolitt and } \\
\text { metamorf tuff }\end{array}$ & Alpine heath & Bulk & Weekly & No & weight of samples \\
\hline Lochnagar & Granite & $\begin{array}{l}\text { Alpine heath, bare } \\
\text { rock }\end{array}$ & Bulk & $\begin{array}{l}\text { Weekly }(\mathrm{S}) \mathrm{Bi}- \\
\text { weekly }(\mathrm{W})\end{array}$ & Yes & rain gauge AWS* \\
\hline Starolesnianske pl. & Granite & Alpine meadows & Bulk & Weekly & Yes & weight of samples \\
\hline Skalnaté pl. & Granite & $\begin{array}{l}\text { Alpine meadows, } \\
\text { bare rock }\end{array}$ & Wet-only & Weekly & No & weight of samples \\
\hline Hala Gasienicowa & $\begin{array}{l}\text { Granitoids and } \\
\text { moraine }\end{array}$ & $\begin{array}{l}\text { Alpine meadows, } \\
\text { dwarf pine }\end{array}$ & Bulk & Weekly & No & weight of samples \\
\hline Gossenköllesee & Granite-Gneiss & Alpine grass heath & Bulk/Wet-only & Weekly & Yes & $\begin{array}{c}\text { meteo station/weight of } \\
\text { samples }\end{array}$ \\
\hline Texel & Acidic rocks & Rocks & Bulk & Every $2 / 3$ mon. & No & weight of samples \\
\hline Renon/Ritten & Quartz-Porphir & Forest (Picea abies) & Bulk/Wet-only & Weekly & No & weight of samples \\
\hline Jörisee & Granite & $\begin{array}{l}\text { Hay meadows, sparse } \\
\text { coniferous plants }\end{array}$ & Bulk/Wet-only & Weekly & Yes & $\begin{array}{c}\text { meteo station/weight of } \\
\text { samples }\end{array}$ \\
\hline Robiei & Cristalline rocks & $\begin{array}{l}\text { Hay meadows and } \\
\text { sparse shrubs }\end{array}$ & Wet-only & Weekly & Yes & $\begin{array}{c}\text { meteo station/weight of } \\
\text { samples }\end{array}$ \\
\hline Graniga & Gneiss & Pasture & Wet-only & Weekly & No & $\begin{array}{c}\text { meteo station/weight of } \\
\text { samples }\end{array}$ \\
\hline Redó & Granite & Grassland & Bulk & Monthly & No & AWS/volume of samples \\
\hline CRAM & Granite & Grassland & $\begin{array}{l}\text { Wet\&dry; bulk } \\
\text { since Jan } 98\end{array}$ & $\begin{array}{c}\text { Daily, single } \\
\text { event since Jan } 98\end{array}$ & No & volume of samples \\
\hline
\end{tabular}

long term variations in the chemistry of some selected lakes will also be considered.

\subsection{Chemical analysis and Analytical Quality Control (AQC)}

Procedures and suggested methods for the chemical analyses were described by Mosello \& Wathne (1997).
Ion chromatography was indicated as the most reliable method for major ion analysis, while molecular spectrophotometry was suggested for the analysis of ammonium (indophenol blue, Fresenius et al. 1988), reactive and total phosphorus (molybden blue, A.P.H.A., A.W.W.A., W.P.C.F., 1992), reactive silica (molibdosilicate reduced to heteropoly blue, A.P.H.A., A.W.W.A., W.P.C.F., 1992), aluminum (pyrocatechol 
Tab. 7. Volume weighted averages of concentrations in atmospheric deposition collected at MOLAR sites (conductivity $\mu \mathrm{S} \mathrm{cm}^{-1}$ at $25^{\circ} \mathrm{C}$; concentrations $\mu$ eq $1^{-1}$ ).

\begin{tabular}{|c|c|c|c|c|c|c|c|c|c|c|c|c|c|c|c|c|c|c|}
\hline Sampling sites & $\begin{array}{l}\text { Starting } \\
\text { date }\end{array}$ & $\begin{array}{l}\text { Ending } \\
\text { date }\end{array}$ & $\begin{array}{c}\text { Volume } \\
\text { (mm) }\end{array}$ & $\mathrm{pH}$ & $\mathrm{H}^{+}$ & $\mathrm{NH}_{4}{ }^{+}$ & $\mathrm{Ca}^{++}$ & $\mathrm{Mg}^{++}$ & $\mathrm{Na}^{+}$ & $\mathrm{K}^{+}$ & Alk & $\mathrm{SO}_{4}=$ & $\mathrm{NO}_{3}^{-}$ & $\mathrm{Cl}^{-}$ & Cat. & An. & $\begin{array}{l}\text { Meas. } \\
\text { cond. }\end{array}$ & $\begin{array}{l}\text { Calc. } \\
\text { cond. }\end{array}$ \\
\hline Kårvatn (W) & 01/07/96 & $30 / 06 / 97$ & 1655 & 5.18 & 7 & 7 & 7 & 19 & 77 & 3 & - & 15 & 4 & 98 & 116 & 115 & 16 & 17 \\
\hline Kårvatn (W) & 01/07/97 & $30 / 06 / 98$ & 1698 & 5.25 & 6 & 8 & 4 & 15 & 64 & 2 & - & 13 & 4 & 76 & 96 & 92 & 14 & 13 \\
\hline Møsvatn (W) & $01 / 07 / 96$ & $30 / 06 / 97$ & 690 & 4.74 & 18 & 14 & 4 & 3 & 9 & 1 & 0 & 18 & 16 & 11 & 49 & 45 & 11 & 11 \\
\hline Møsvatn (W) & $01 / 07 / 97$ & $30 / 06 / 98$ & 746 & 4.72 & 19 & 12 & 3 & 1 & 5 & 1 & 0 & 19 & 16 & 6 & 40 & 42 & 7 & 6 \\
\hline Lochnagar (W) & $29 / 08 / 96$ & $30 / 06 / 97$ & 1426 & 4.79 & 16 & 20 & 14 & 13 & 39 & 3 & - & 28 & 18 & 39 & 106 & 84 & 16 & 17 \\
\hline Lochnagar (W) & $01 / 07 / 97$ & $30 / 06 / 98$ & 1627 & 4.64 & 23 & 19 & 10 & 12 & 35 & 2 & - & 31 & 17 & 35 & 100 & 83 & 22 & 19 \\
\hline Starolesnianske pl. (B) & $15 / 08 / 96$ & $30 / 06 / 97$ & 851 & 4.42 & 38 & 24 & 11 & 2 & 4 & 1 & 0 & 53 & 19 & 6 & 81 & 79 & 22 & 22 \\
\hline Starolesnianske pl. (B) & $01 / 07 / 97$ & $30 / 06 / 98$ & 1302 & 4.55 & 28 & 24 & 12 & 3 & 7 & 3 & 0 & 50 & 17 & 7 & 78 & 75 & 19 & 19 \\
\hline Skalnaté pl. (W) & $08 / 11 / 96$ & $30 / 06 / 97$ & 485 & 4.39 & 41 & 29 & 14 & 3 & 3 & 1 & 0 & 54 & 24 & 5 & 92 & 84 & 21 & 24 \\
\hline Skalnaté pl. (W) & 01/07/97 & $30 / 06 / 98$ & 1169 & 4.49 & 33 & 18 & 9 & 3 & 3 & 1 & 0 & 41 & 15 & 3 & 65 & 60 & 17 & 18 \\
\hline Hala Gasienicowa (B) & $01 / 07 / 96$ & 01/06/97 & 1684 & - & - & 24 & 16 & 5 & 4 & - & 0 & 79 & 28 & - & 124 & 165 & 22 & 32 \\
\hline Hala Gasienicowa (B) & $01 / 07 / 97$ & 01/06/98 & 1829 & -0 & - & 13 & 8 & 3 & 6 & - & 0 & 28 & 23 & - & 122 & 69 & 21 & 25 \\
\hline Gossenköllesee (W) & $01 / 10 / 96$ & $30 / 06 / 97$ & 877 & 5.05 & 9 & 11 & 6 & 3 & 3 & 0 & 1 & 11 & 13 & 3 & 32 & 28 & 7 & 6 \\
\hline Gossenköllesee (W) & $01 / 07 / 97$ & $30 / 06 / 98$ & 979 & 4.97 & 11 & 15 & 7 & 2 & 2 & 1 & 2 & 13 & 13 & 3 & 38 & 31 & 8 & 7 \\
\hline Texel (B) & $17 / 07 / 96$ & $14 / 07 / 97$ & 898 & 5.30 & 5 & 45 & 10 & 5 & 4 & 2 & 8 & 33 & 25 & 6 & 72 & 73 & 13 & 12 \\
\hline Texel (B) & $15 / 07 / 97$ & $22 / 06 / 98$ & 729 & 5.11 & 8 & 40 & 16 & 9 & 3 & 6 & 21 & 35 & 32 & 8 & 81 & 95 & 15 & 14 \\
\hline Ritten (B) & $01 / 07 / 96$ & $30 / 06 / 97$ & 1189 & 5.20 & 6 & 27 & 12 & 5 & 7 & 12 & 13 & 24 & 23 & 8 & 69 & 67 & 11 & 11 \\
\hline Ritten (B) & $01 / 07 / 97$ & $30 / 06 / 98$ & 853 & 5.27 & 5 & 36 & 16 & 8 & 6 & 13 & 17 & 28 & 26 & 9 & 85 & 81 & 13 & 13 \\
\hline Ritten (W) & $01 / 07 / 96$ & $30 / 06 / 97$ & 1265 & 5.07 & 8 & 23 & 11 & 4 & 4 & 2 & 5 & 23 & 19 & 7 & 53 & 54 & 10 & 10 \\
\hline Ritten (W) & $01 / 07 / 97$ & $30 / 06 / 98$ & 820 & 5.17 & 7 & 26 & 13 & 7 & 4 & 3 & 6 & 25 & 20 & 7 & 60 & 58 & 10 & 10 \\
\hline Jörisee (B) & $12 / 07 / 96$ & 03/10/96 & 405 & 5.02 & 10 & 9 & 13 & 2 & 6 & 4 & 6 & 19 & 21 & 8 & 44 & 54 & 10 & 8 \\
\hline Jörisee (S core) & $17 / 02 / 97$ & & 326 & 5.67 & 2 & 2 & 2 & 0 & 1 & 0 & 1 & 2 & 4 & 1 & 7 & 8 & 3 & 2 \\
\hline Jörisee (B) & $23 / 07 / 97$ & $17 / 10 / 97$ & 295 & 5.01 & 10 & 7 & 20 & 4 & 9 & 7 & - & 22 & 19 & 11 & 57 & 51 & 15 & 13 \\
\hline Jörisee (S core) & $10 / 03 / 98$ & & 324 & 4.98 & 10 & 3 & 3 & 1 & 1 & 0 & 0 & 3 & 7 & 2 & 18 & 12 & 5 & 5 \\
\hline Robiei (W) & $01 / 07 / 96$ & $30 / 06 / 97$ & 2401 & 5.02 & 9 & 14 & 9 & 3 & 2 & 1 & 0 & 24 & 12 & 3 & 38 & 39 & 7 & 8 \\
\hline Robiei (W) & $01 / 07 / 97$ & $30 / 06 / 98$ & 2221 & 5.05 & 9 & 16 & 15 & 3 & 4 & 1 & 5 & 20 & 15 & 3 & 46 & 43 & 8 & 9 \\
\hline Graniga (W) & $01 / 07 / 96$ & 01/06/97 & 1885 & 4.67 & 21 & 34 & 10 & 2 & 5 & 2 & 1 & 32 & 28 & 5 & 75 & 66 & 17 & 16 \\
\hline Graniga (W) & $01 / 07 / 97$ & 01/06/98 & 846 & 4.70 & 20 & 22 & 9 & 3 & 4 & 3 & 1 & 24 & 24 & 4 & 61 & 53 & 14 & 14 \\
\hline Redó (W, B) & $30 / 06 / 97$ & $30 / 06 / 98$ & 1381 & 4.97 & 11 & 17 & 20 & 3 & 13 & 3 & 0 & 22 & 11 & 11 & 66 & 49 & 10 & 10 \\
\hline
\end{tabular}

violet, ISO 10566: 1994) and aluminum fractions (Rogeberg \& Henriksen 1985).

As part of the AQC within each laboratory, checking the ion balance and comparing measured and calculated conductivity were suggested as a routine operation for all the analyses performed. The same checks were repeated on the whole set of data by the two co-ordinating laboratories (surface water: NIVA, atmospheric deposition: CNR). They were performed using an MS-Excel sheet, distributed to all laboratories, and also used for mailing the data from each laboratory to the co-ordinating centres.

Inter-laboratory exercises were carried out for the whole period of the project to assure the comparability of the results produced by each institution. Two different intercomparison networks were used: the first referred to the circuit of the ICP on Assessment and Monitoring of Acidification of Rivers and Lakes (ICP Waters), co-ordinated by NIVA, the second to the circuit of the Analytical Quality Control and Assessment Studies in the Mediterranean Basin (AQUACON), carried out by the CNR in collaboration with the Environment Institute of the Joint Research Centre at Ispra. Results were published in reports (e.g., Hovind 1997; Mosello et al. 1998a, b) and discussed in an annual technical workshop. Besides their function in checking the comparability of the results, these exercises provide the possibility for exploring specific analytical problems, such as the measurement of $\mathrm{pH}$ and low values of alkalinity, both variables important to the aims of the project (Mosello \& Wathne 1997; Marchetto et al. 1997).

\section{RESULTS}

\subsection{Atmospheric deposition}

Some of the sampling stations were not continuously operational for the whole of the two year period (July 96-June 98), due to various problems, mainly linked to difficulties in reaching the sites. The period considered for each station is indicated in table 7, which also reports the precipitation volumes and the mean volume weighted concentration of the main ions, separately for the two study years. The values refer both to rain and snow deposition, with the aim of evaluating the total ion deposition. Even in the stations which worked regularly for the whole period, the volumes of precipitation show considerable differences in the two study years; for example, Graniga (Central Alps), has annual volumes of 1885 and $846 \mathrm{~mm}$.

The analytical quality of the data was generally good, as emerges from the ion balances and from the comparison between measured and calculated conductivity (Tab. 7). The $\mathrm{pH}$ values range from 4.1-4.5 in the Tatra Mountains (Starolesnianske pleso, Skalnaté pleso, Hala Gasienicowa sampling sites), to 4.7-5.0 in the other sites. Sulphate and nitrate prevail among the ani- 
ons in most of the stations, with the exception of Kårvatn and Lochnagar, which have a marked contribution of chloride of marine origin. The sulphate to nitrate ratio is close to 2 in the stations in the Tatra Mountains and Spain, while it approaches 1 in the sites in the Alps and in Møsvatn (Central part of Southern Norway). The higher ratio for the Tatra Mountains is probably due to higher sulphur emissions deriving from fuel combustion. Even the frequency of dust deposition events, more frequent in Southern Europe, could affect the mean annual sulphate concentration, as these events are characterised by high sulphate content (Carratala et al. 1996).

Alkalinity was measured in significant amounts in some stations in the Eastern Alps (Ritten, Texel). Its deposition is mainly related to the transport of Saharan dust (Guerzoni \& Chester 1996), which happens occasionally (from 2 to 5 times each year), but with high precipitation volumes in most cases, so that they are important in determining the annual volume weighted values.

Among cations, the hydrogen ion prevails in the stations with $\mathrm{pH}$ lower than 4.5. Ammonium and calcium are the most important ions in the other stations, with the exception of the two sites close to the sea, where the highest concentrations are of sodium.

The total ion content ranges between $60 \mu \mathrm{eq} 1^{-1}$ in Gossenköllesee (Eastern Alps) and $200 \mu \mathrm{eq} \mathrm{l^{-1 }}$ for the stations of Kårvatn (Norway) and Lochnagar (Scotland), both with a high contribution of sodium and chloride of marine origin

The comparability of the data (Fig. 2) improves if they are corrected for seasalt, assuming that all chloride is of marine origin and that the ratio with the other ions $\left(\mathrm{Na}^{+}, \mathrm{Mg}^{++}, \mathrm{Ca}^{++}, \mathrm{SO}_{4}{ }^{\circ}\right)$ is the same as in seawater. Results (Fig. 2b) show that the lowest ion concentrations were then found in Kårvatn $\left(25 \mu \mathrm{eq} \mathrm{l}^{-1}\right)$, while in the other stations the correction does not substantially affect the concentrations of sulphate and the sulphate to nitrate ratio.

Relationships among ions in deposition were tested for each sampling site using cluster analysis. Results showed marked similarities in the grouping of ions, so that four examples were chosen, on the basis of (a) strong influence of marine salts (Lochnagar), (b) high acidity (Skalnaté pleso) (c) low acidity in the Alps (Ritten) and (d) in the Pyrenees (Redó) (Fig. 3). All the stations show a strong correlation between sodium and chloride, due to the fact that most of these ions are from sea-spray, and a grouping of $\mathrm{SO}_{4}{ }^{=}, \mathrm{NH}_{4}{ }^{+}, \mathrm{NO}_{3}{ }^{-}$, i.e. the ions mainly deriving from anthropogenic activity. The hydrogen ion concentration is directly linked to the group of "acid" compounds at Lochnagar, Skalnaté pleso and Redó, while in the less acidic deposition of Ritten, $\mathrm{H}^{+}$is correlated to the group of ions of marine origin.

Deposition values show a more marked yearly variation if compared with concentrations, mainly due to the different amounts of precipitation. Some of the stations were not operational for the whole two year period, so that the amount of precipitation measured refers only to fractions of the year. For these reasons the wet deposition of ions is presented in table 8 as mean values for the two years of study, and in figure 4, where the depositions are also shown without the marine contribution.
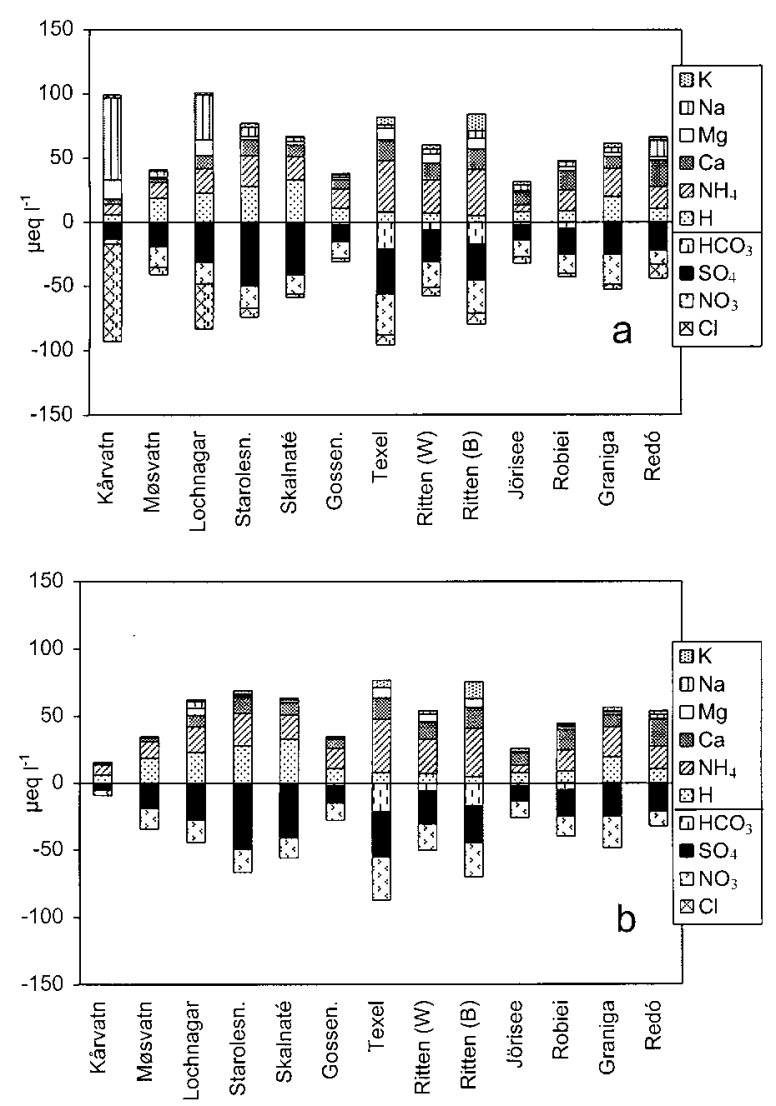

Fig. 2. Ion balance for the mean annual concentration of atmospheric deposition before (a) and after (b) sea-salt correction.

The stations in the Alps show wide variability in the deposition of acidity, from relatively high values (20-30 meq $\mathrm{m}^{-2} \mathrm{y}^{-1}$ for the sites of Graniga and Robiei in the Western Alps at altitudes between 1100-1900 m a.s.l) to the lowest values of 9-10 meq $\mathrm{m}^{-2} \mathrm{y}^{-1}$ for the stations of Gossenköllesee and Jörisee, both located at about 2500 $\mathrm{m}$ a.s.l. The load of hydrogen ion is also low in the Alpine station of Ritten (1780 $\mathrm{m}$ a.s.1.), in the Eastern Southern Alps. In these stations the ammonium load is high compared with the hydrogen ion load, and the oxidation of ammonium to nitrate may be a further source of acidity. The situation is better in the case of the northermost stations of Kårvatn and Møsvatn, where both hydrogen and ammonium loads are low (Tab. 8). 
Lochnagar

Ward's method - 1 Pearson
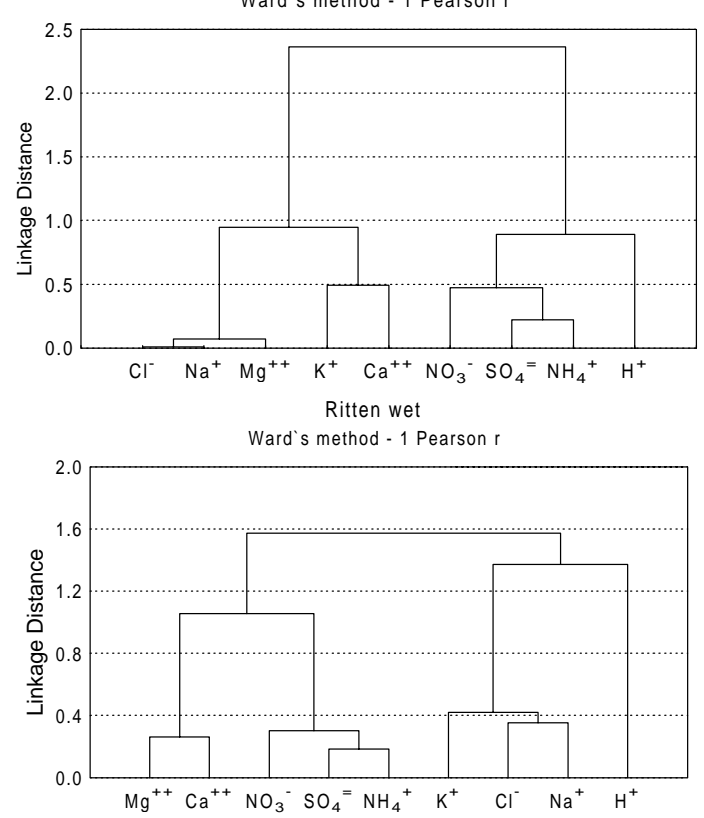

Skalnatè wet

Ward's method - 1 Pearson $r$

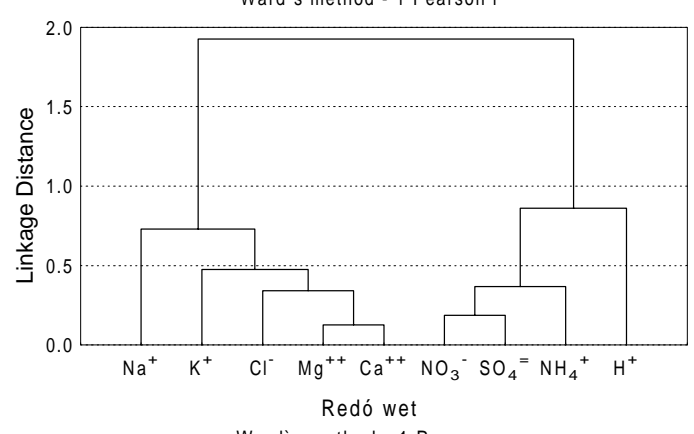

Ward's method - 1 Pearson $r$

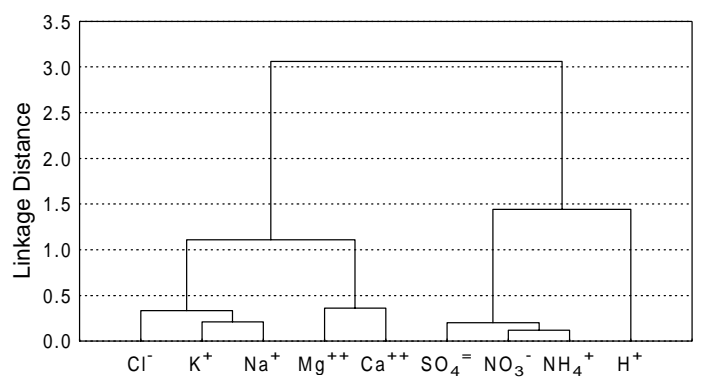

Fig. 3. Cluster analysis of the ion concentrations in atmospheric deposition of four sampling stations.

As regards the marine contributions of sodium and chloride, it is consistent with the concentration values that the highest depositions are found in the stations of Kårvatn and Lochnagar, both close to the sea.

The base cation deposition of non-marine origin is mainly represented by calcium. The highest deposition values were found in the stations of Robiei and Redó. In Robiei, it was determined by several episodes of Saharan dust deposition, whereas in Redó it represented background values, since no acute dust deposition events were recorded. In years in which such episodes occur, the calcium deposition may triplicate (Camarero \& Catalan 1993).

\subsection{Lakes}

The mean concentrations for surface water in the MOLAR lakes are shown in table 9, where the number of samplings performed in each site is also indicated. The ion concentrations have quite a wide range of variation (88-3360 meq $\mathrm{l}^{-1}$ ), from very diluted to highly buffered waters. The corresponding range of variation of conductivity is $6-148 \mu \mathrm{S} \mathrm{cm}^{-1}$ at $25{ }^{\circ} \mathrm{C}$. The $\mathrm{pH}$ values are in the range 4.9-6.0 in 8 of the 23 lakes, while three lakes (La Caldera, Hagelsee and Jezero v Ledvici) show values higher than 7.5. Alkalinity is very high for Jezero v Ledvici and Hagelsee (1303 and 1167 $\mu$ eq $\mathrm{l}^{-1}$ ), ranges between 118-343 $\mu$ eq $\mathrm{l}^{-1}$ for lakes Jörisee, Saanajärvi and La Caldera, and is lower than 50 and $20 \mu \mathrm{eq}^{-1}$ in 15 and 7 lakes, respectively.
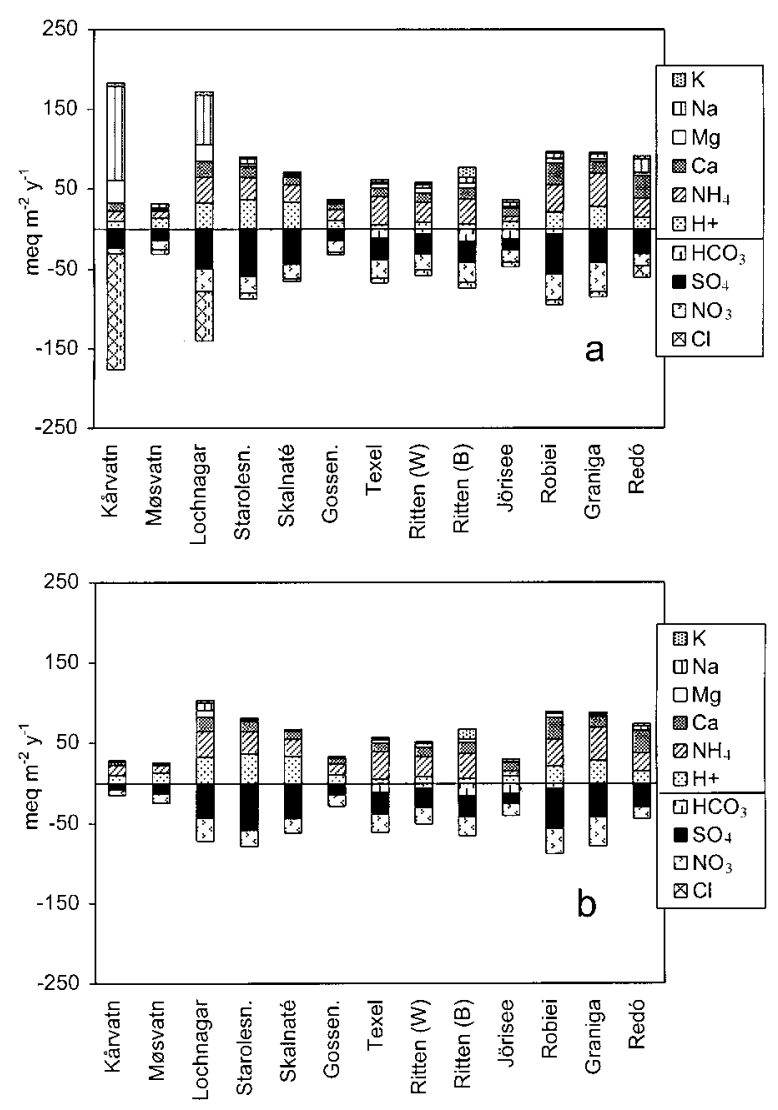

Fig. 4. Deposition of ions from atmospheric deposition before (a) and after (b) sea-salt correction. 
Tab. 8. Deposition of ions (meq $\mathrm{m}^{-2} \mathrm{y}^{-1}$ ) from atmospheric deposition.

\begin{tabular}{|c|c|c|c|c|c|c|c|c|c|c|c|c|c|c|}
\hline Sampling sites & Starting date & Ending date & $\mathrm{H}^{+}$ & $\mathrm{NH}_{4}^{+}$ & $\mathrm{Ca}^{++}$ & $\mathrm{Mg}^{++}$ & $\mathrm{Na}^{+}$ & $\mathrm{K}^{+}$ & Alk & $\mathrm{SO}_{4}=$ & $\mathrm{NO}_{3}{ }^{-}$ & $\mathrm{Cl}^{-}$ & Cations & Anions \\
\hline Kårvatn (W) & 01/07/96 & $30 / 06 / 98$ & 10 & 13 & 9 & 29 & 118 & 5 & 0 & 23 & 7 & 146 & 178 & 173 \\
\hline Møsvatn (W) & 01/07/96 & $30 / 06 / 98$ & 13 & 9 & 2 & 1 & 5 & 1 & 0 & 14 & 12 & 6 & 25 & 19 \\
\hline Lochnagar (W) & 29/08/96 & $30 / 06 / 98$ & 33 & 32 & 20 & 21 & 62 & 4 & 0 & 49 & 29 & 62 & 172 & 140 \\
\hline Starolesnianske pl.(B) & $15 / 08 / 96$ & $30 / 06 / 98$ & 37 & 27 & 14 & 3 & 7 & 2 & 0 & 59 & 21 & 8 & 90 & 89 \\
\hline Skalnaté pl.(W) & 08/11/96 & $30 / 06 / 98$ & 34 & 21 & 10 & 2 & 3 & 1 & 0 & 44 & 18 & 4 & 71 & 66 \\
\hline Hala Gasienicowa (B) & $01 / 07 / 96$ & $01 / 06 / 98$ & 120 & 32 & 21 & 7 & 9 & - & 0 & 92 & 45 & - & 166 & 173 \\
\hline Gossenköllesee (W) & 01/10/96 & $30 / 06 / 98$ & 10 & 14 & 7 & 2 & 3 & 0 & 2 & 12 & 14 & 3 & 36 & 28 \\
\hline Texel (B) & $17 / 07 / 96$ & 22/06/98 & 5 & 35 & 10 & 5 & 3 & 3 & 11 & 27 & 23 & 6 & 62 & 67 \\
\hline Ritten (B) & 01/07/96 & $30 / 06 / 98$ & 6 & 31 & 14 & 7 & 7 & 13 & 15 & 27 & 25 & 8 & 77 & 74 \\
\hline Ritten (W) & $01 / 07 / 96$ & $30 / 06 / 98$ & 8 & 25 & 12 & 6 & 5 & 3 & 6 & 24 & 21 & 7 & 58 & 58 \\
\hline Jörisee (B) & $12 / 07 / 96$ & $10 / 03 / 98$ & 9 & 6 & 11 & 2 & 5 & 3 & 0 & 14 & 15 & 6 & 37 & 37 \\
\hline Robiei (W) & $01 / 07 / 96$ & $30 / 06 / 98$ & 21 & 34 & 27 & 6 & 7 & 2 & 6 & 50 & 32 & 6 & 97 & 95 \\
\hline Graniga (W) & 01/07/96 & $01 / 06 / 98$ & 29 & 41 & 14 & 3 & 6 & 3 & 1 & 41 & 37 & 7 & 96 & 85 \\
\hline Redó (W, B) & $30 / 06 / 97$ & $30 / 06 / 98$ & 15 & 23 & 28 & 4 & 18 & 4 & 0 & 30 & 15 & 15 & 91 & 68 \\
\hline
\end{tabular}

Tab. 9. Mean chemical characteristics of MOLAR lakes (conductivity $\mu \mathrm{S} \mathrm{cm}^{-1}$ at $25^{\circ} \mathrm{C}$; concentrations $\mu$ eq $1^{-1}$ ).

\begin{tabular}{|c|c|c|c|c|c|c|c|c|c|c|c|c|c|c|c|c|c|}
\hline Lake name & $\mathrm{n}^{\circ}$ data & $\mathrm{pH}$ & $\mathrm{H}^{+}$ & $\mathrm{NH}_{4}^{+}$ & $\mathrm{Ca}^{++}$ & $\mathrm{Mg}^{++}$ & $\mathrm{Na}^{+}$ & $\mathrm{K}^{+}$ & Alk & $\mathrm{SO}_{4}=$ & $\mathrm{NO}_{3}^{-}$ & $\mathrm{Cl}^{-}$ & Cations & Anions & $\Sigma$ Ions & Meas. cond. & Calc. cond. \\
\hline Øvre Neådalsvatn & 93 & 6.22 & 1 & 0 & 32 & 14 & 44 & 4 & 32 & 17 & 2 & 47 & 94 & 98 & 193 & 11.5 & 11.9 \\
\hline Stavsvatn & 15 & 5.93 & 1 & 2 & 44 & 10 & 21 & 5 & 19 & 35 & 7 & 17 & 82 & 78 & 160 & 10.4 & 10.5 \\
\hline Lochnagar & 71 & 5.32 & 5 & 1 & 40 & 41 & 93 & 6 & 8 & 55 & 19 & 80 & 186 & 162 & 348 & 22.0 & 23.7 \\
\hline Lago Paione Superiore & 24 & 5.82 & 2 & 3 & 42 & 8 & 11 & 7 & 7 & 35 & 26 & 5 & 72 & 73 & 146 & 10.7 & 10.2 \\
\hline Lago Paione Inferiore & 15 & 6.55 & 0 & 0 & 68 & 11 & 14 & 10 & 34 & 44 & 25 & 4 & 103 & 106 & 209 & 13.1 & 13.2 \\
\hline Lago di Latte & 15 & 6.46 & 1 & 4 & 100 & 12 & 16 & 8 & 70 & 59 & 17 & 3 & 141 & 148 & 290 & 18.3 & 17.8 \\
\hline Aubè & 1 & 5.71 & 2 & 0 & 24 & 6 & 13 & 3 & 6 & 20 & 8 & 6 & 48 & 40 & 88 & 6.1 & 6.2 \\
\hline Arresjøen & 2 & 6.41 & 0 & 1 & 35 & 52 & 216 & 6 & 26 & 34 & 1 & 251 & 311 & 311 & 622 & 38.5 & 39.4 \\
\hline Schwarzsee ob Sölden & 3 & 5.98 & 1 & 1 & 80 & 28 & 19 & 4 & 8 & 106 & 8 & 3 & 132 & 125 & 257 & 16.7 & 17.5 \\
\hline Gossenköllesee & 38 & 6.76 & 0 & 1 & 142 & 18 & 15 & 5 & 86 & 58 & 19 & 3 & 181 & 166 & 346 & 19.2 & 20.7 \\
\hline Redó & 23 & 6.41 & 0 & 1 & 72 & 7 & 11 & 1 & 47 & 27 & 12 & 7 & 92 & 94 & 186 & 11.3 & 11.2 \\
\hline La Caldera & 21 & 8.12 & 0 & 1 & 241 & 54 & 14 & 2 & 343 & 18 & 12 & 9 & 311 & 382 & 693 & 29.3 & 36.3 \\
\hline Starolesnianske pl. & 49 & 4.95 & 12 & 4 & 28 & 6 & 7 & 2 & -3 & 43 & 13 & 5 & 59 & 58 & 116 & 11.8 & 11.5 \\
\hline Nižné Terianske pl. & 16 & 6.48 & 0 & 1 & 150 & 8 & 14 & 3 & 77 & 55 & 36 & 5 & 177 & 173 & 350 & 21.2 & 21.3 \\
\hline Długi Staw & 17 & 5.59 & 5 & 9 & 92 & 12 & 14 & 3 & 42 & 56 & 42 & 11 & 135 & 151 & 286 & 26.5 & 19.7 \\
\hline Laguna Cimera & 25 & 6.33 & 1 & 2 & 17 & 11 & 13 & 2 & 31 & 11 & 4 & 6 & 46 & 51 & 97 & 5.7 & 5.7 \\
\hline z. Chuna & 22 & 6.14 & 1 & 0 & 46 & 11 & 24 & 2 & 20 & 35 & 4 & 21 & 85 & 81 & 165 & 10.3 & 10.6 \\
\hline Jezero v Ledvici & 34 & 7.69 & 0 & 5 & 1199 & 639 & 42 & 6 & 1303 & 42 & 20 & 105 & 1891 & 1470 & 3361 & 148.2 & 179.0 \\
\hline Limgambergtjern & 4 & 5.79 & 2 & 1 & 56 & 51 & 122 & 4 & 12 & 79 & 0 & 129 & 236 & 220 & 456 & 28.2 & 29.9 \\
\hline Saanajärvi & 27 & 7.02 & 0 & 1 & 145 & 51 & 51 & 6 & 175 & 86 & 3 & 49 & 256 & 314 & 569 & 27.5 & 33.2 \\
\hline Hagelsee & 23 & 8.11 & 0 & 4 & 1225 & 205 & 21 & 14 & 1167 & 252 & 12 & 18 & 1468 & 1449 & 2917 & 115.0 & 160.4 \\
\hline Jörisee & 28 & 7.03 & 0 & 1 & 128 & 27 & 18 & 9 & 118 & 56 & 17 & 3 & 183 & 193 & 376 & 17.4 & 21.8 \\
\hline Laghetto Inf. & 12 & 6.56 & 0 & 1 & 64 & 9 & 12 & 10 & 34 & 38 & 16 & 2 & 96 & 90 & 187 & 11.6 & 11.7 \\
\hline
\end{tabular}

Sulphate ranges from 11 to $252 \mu$ eq $1^{-1}$; the highest values show that atmospheric deposition cannot be considered as the only source of sulphate, but in some cases (e.g., Hagelsee, Jezero v Ledvici, Schwarzsee ob Sölden) there is also a sulphate contribution deriving from watershed weathering. Nitrate concentrations are very low (below $10 \mu \mathrm{eq}^{-1}$ ) in the northernmost lakes in Norway (Øvre Neådalsvatn, Stavsvatn, Limgambergtjern and Arresjøen), Finland (Saanajärvi), Russia (z. Chuna) and Spain (Laguna Cimera), while the highest values (36-42 $\mu \mathrm{eq}^{-1}$ ) are found in the lakes in the Tatra Mountains (N. Terianske pl. and Długi Staw). The lakes in the Alps show values between 15-26 $\mu$ eq $1^{-1}$, with the lowest value for Hagelsee $\left(12 \mu \mathrm{eq}^{-1}\right)$. Nitrate concentration is also relatively low (below $12 \mu \mathrm{eq} \mathrm{l}^{-1}$ ) in the lakes in Spain (Redó, Cimera and La Caldera).

A comparison between the different lakes is possible on the basis of the results of the cluster analysis (Fig. 5), performed on the concentrations of the main ions, lognormalised and using Ward's method and the Euclidean distances as clustering criteria. Four main groups emerge; the main chemical characteristics of each group are given in figure 6 . The first group comprises two lakes (Hagelsee and Jezero v Ledvici), characterised by high alkalinty values, calcium and magnesium concentrations. The mean total ion concentration is $3140 \mu \mathrm{eq}$ $1^{-1}$, with a conductivity of $170 \mu \mathrm{S} \mathrm{cm}^{-1}$. The chemistry of the lakes of the second group (4 lakes: Limgambergtjern, Arresjøen, Saanajärvi, Lochnagar) is characterised by a lower ion concentration $\left(500 \mu \mathrm{eq} \mathrm{l}^{-1}\right.$, conductivity of $32 \mu \mathrm{S} \mathrm{cm}^{-1}$ ) and by the importance in the ionic balance of sodium, chloride, calcium and sulphate, which altogether represent up to $70 \%$ of the total ionic concentrations. Groups 3 and 4 (11 and 6 lakes, respectively) show minor differences, the most important being the lower ionic concentrations in group 4 (148 $\mu$ eq 
$1^{-1} v s 296 \mu$ eq $1^{-1}$ of group 3), the higher nitrate and sulphate concentrations in group 3 (21 and $52 \mu \mathrm{eq} \mathrm{l}^{-1}$ respectively vs 6 and $24 \mu \mathrm{eq} 1^{-1}$ of group 4) and the higher concentrations in sodium and chloride in group 4 (21 and $17 \mu$ eq $\mathrm{l}^{-1}$ respectively vs 14 and $5 \mu \mathrm{eq} \mathrm{l}^{-1}$ of group 3).

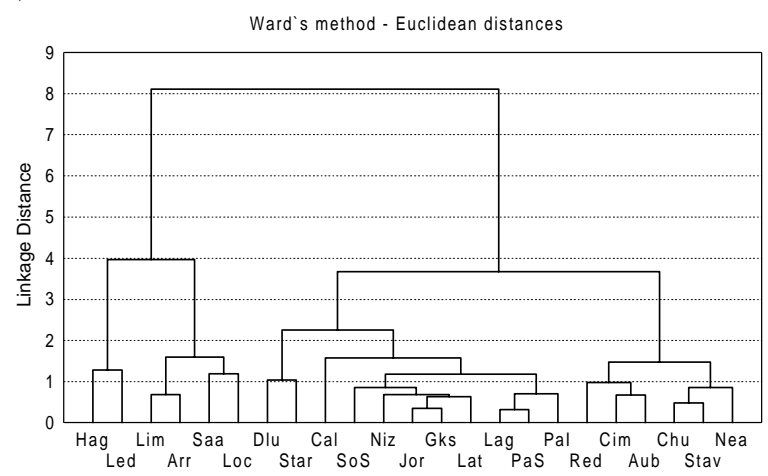

Fig. 5. Cluster analysis of the chemistry of MOLAR lakes.
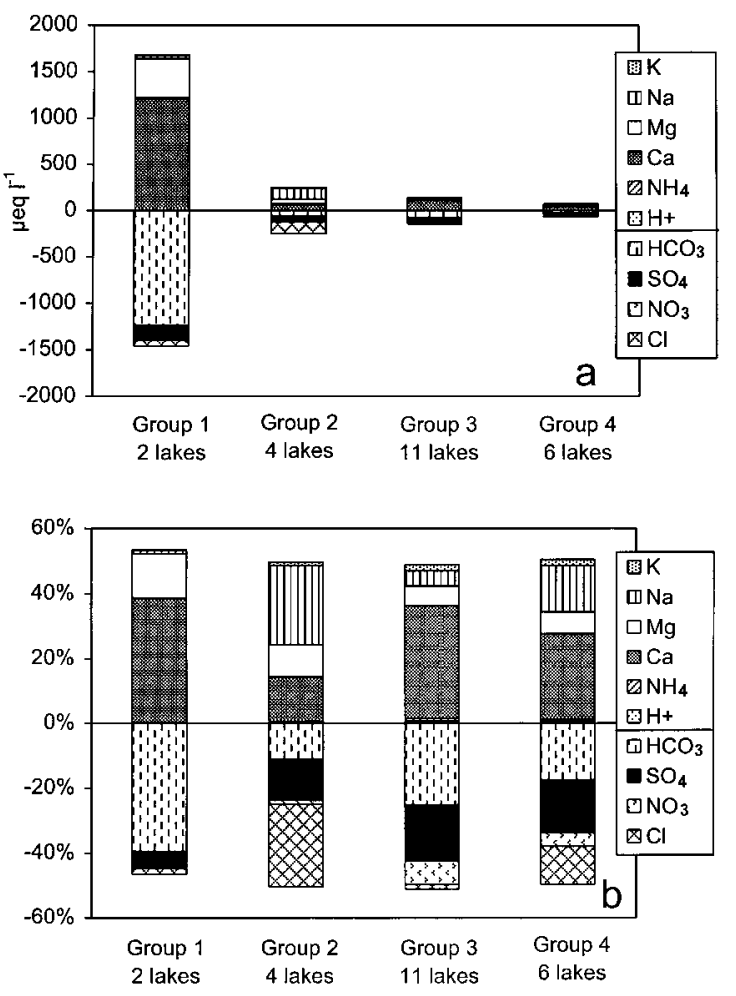

Fig. 6. Mean chemical characteristics of the four clusters of the MOLAR lakes.

\section{DISCUSSION}

\subsection{Bulk vs wet-only chemistry of atmospheric deposition}

The type of sampling employed in determining the chemistry of atmospheric deposition is of major importance. Wet deposition should be measured using wetonly samplers, i.e. funnels which are open to the atmos- phere only during precipitation events. Continuously open funnels (bulk samplers) were used in some stations where no power source was available. Several studies considering the ratio between bulk and wet-only chemistry have shown considerable variability (Draajers \& Erisman 1993; Van Leeuwen et al. 1996). A comparison of 40 annual or pluriannual studies carried out in different European sites shows a mean wet/bulk ratio between $0.67 \pm 0.16$ and $0.73 \pm 0.16$ for $\mathrm{K}, \mathrm{Ca}$ and $\mathrm{Mg}$, of between $0.81 \pm 0.17$ and $0.89 \pm 0.17$ for $\mathrm{Na}^{+}, \mathrm{Cl}^{-}, \mathrm{NO}_{3}^{-}, \mathrm{SO}_{4}{ }^{=}$, $\mathrm{NH}_{4}{ }^{+}$, while the ratio was 1.03 for hydrogen ion (Draajers et al. 1998).

In one of the MOLAR stations in the Alps (Ritten), both bulk and wet-only samples were collected during the whole study period. A comparison of the results, for both concentration and deposition, shows wet/bulk ratios in the same range as that indicated above, with the exception of potassium, where the contribution due to dust is much higher and the ratio falls as low as 0.2.

A further study was made in the Pyrenees, at Redó, by Camarero \& Catalan (1996). The volume of precipitation collected in the period July 1997-January 1998 was $966 \mathrm{~mm}$, with the precipitation occurring on 137 days as opposed to 83 dry days. Dry deposition, expressed as a percentage of bulk (wet+dry) deposition, varied among the elements: from 5-6\% in the case of $\mathrm{NO}_{3}{ }^{-}$and $\mathrm{SO}_{4}{ }^{-}$, to $11-14 \%$ for $\mathrm{NH}_{4}{ }^{+}, \mathrm{Ca}^{++}$and $\mathrm{Mg}^{++}, 17-$ $19 \%$ for $\mathrm{Cl}^{-}$and $\mathrm{Na}^{+}$, and up to $35 \%$ for $\mathrm{K}^{+}$. Assuming that the difference between bulk and total deposition is not relevant to the purpose of this discussion, the ratios of wet/total deposition are still in the range indicated above (Tab. 10).

These results suggest certain characteristics of the transport and deposition of the different ions:

- the sum of $\mathrm{NO}_{3}{ }^{-}$and $\mathrm{SO}_{4}{ }^{=}$(in equivalents) practically equals $\mathrm{NH}_{4}{ }^{+}$in wet deposition, indicating that $\mathrm{NO}_{3}\left(\mathrm{NH}_{4}\right)$ and $\mathrm{SO}_{4}\left(\mathrm{NH}_{4}\right)_{2}$ aerosols are the main source of these ions. Ammonium aerosols seem to be very efficiently scavenged by precipitation as condensation nucleii: only about $5 \%$ of both $\mathrm{NO}_{3}{ }^{-}$ and $\mathrm{SO}_{4}{ }^{2}$ is deposited as dry deposition. Dry deposition of $\mathrm{NH}_{4}{ }^{+}$is approximately double (c. 12\%), which suggests that other mechanisms of dry deposition, gaseous $\mathrm{NH}_{3}$ for instance, may be of the same order as that of ammonium aerosols;

- $\mathrm{Cl}^{-}$and $\mathrm{Na}^{+}$have an ionic ratio very close to 1 , in both wet and dry deposition. This indicates that aerosols originating as sea spray are the main mechanism of transport, and are deposited mainly as condensation nucleii in wet deposition (c. 80\%), but also by settling as dry deposition in a significant fraction (c. 20\%);

- $\quad$ as for the other cations, $\mathrm{K}^{+}$presents the highest dry deposition rate (35\%), although the absolute amounts are the lowest compared to the other ions. The relative dry deposition of $\mathrm{Ca}^{++}$and $\mathrm{Mg}^{++}$is much lower (13\%), indicating that the long range 
Tab. 10. Comparison of wet and dry deposition $\left(\mathrm{meq}^{-2}\right)$ collected at Lake Redó site during the study period.

\begin{tabular}{lcccccccccc}
\hline & $\begin{array}{c}\text { Precipitation } \\
(\mathrm{mm})\end{array}$ & $\begin{array}{c}\text { Occurrence } \\
\text { (days) }\end{array}$ & $\mathrm{NH}_{4}^{+}$ & $\mathrm{SO}_{4}{ }^{-}$ & $\mathrm{NO}_{3}^{-}$ & $\mathrm{Cl}^{-}$ & $\mathrm{Ca}^{++}$ & $\mathrm{Mg}^{++}$ & $\mathrm{Na}^{+}$ & $\mathrm{K}^{+}$ \\
\hline Wet-only & 966 & 137 & 17 & 7.7 & 10.2 & 8.5 & 21.2 & 2.7 & 9 & 1.6 \\
Dry & - & 83 & 2.3 & 0.4 & 0.6 & 2.0 & 2.7 & 0.4 & 1.8 & 0.9 \\
Total & - & - & 19.3 & 8.1 & 10.8 & 10.5 & 23.9 & 3.1 & 10.8 & 2.5 \\
Ratio wet/total & - & - & 0.88 & 0.95 & 0.94 & 0.81 & 0.89 & 0.87 & 0.83 & 0.64 \\
\hline
\end{tabular}

Tab. 11. Comparison of water equivalent and ionic loads in precipitation (wet only) and in the snow cover (Pit 1 and Pit 2) at Gossenköllesee, winter 1997/98.

\begin{tabular}{|c|c|c|c|c|c|c|c|c|c|c|c|c|}
\hline \multirow[b]{2}{*}{ Date } & \multicolumn{3}{|c|}{ Water equivalent $(\mathrm{cm})$} & \multicolumn{3}{|c|}{$\mathrm{NO}_{3}^{-}\left(\right.$meq m$\left.{ }^{-2}\right)$} & \multicolumn{3}{|c|}{$\mathrm{NH}_{4}{ }^{+}\left(\right.$meq m$\left.{ }^{-2}\right)$} & \multicolumn{3}{|c|}{$\mathrm{SO}_{4}=\left(\right.$ meq m$\left.{ }^{-2}\right)$} \\
\hline & Wet only & Pit 1 & Pit 2 & Wet only & Pit 1 & Pit 2 & Wet only & Pit 1 & Pit 2 & Wet only & Pit 1 & Pit 2 \\
\hline $30 / 12 / 97$ & 10 & 18 & 39 & 0.78 & 1.23 & 2.46 & 0.34 & 0.23 & 0.52 & 0.45 & 0.52 & 1.08 \\
\hline $27 / 01 / 98$ & 16 & 26 & 35 & 1.21 & 1.99 & 2.28 & 0.58 & 0.21 & 0.59 & 0.75 & 0.88 & 0.93 \\
\hline $24 / 02 / 98$ & 17 & 35 & 41 & 1.48 & 3.08 & 2.67 & 0.79 & 1.11 & 0.80 & 0.92 & 1.73 & 1.42 \\
\hline $11 / 03 / 98$ & 22 & 42 & 38 & 1.90 & 3.63 & 2.99 & 1.11 & 1.11 & 1.49 & 1.31 & 1.76 & 1.54 \\
\hline 08/04/98 & 28 & 45 & 42 & 3.44 & 5.94 & 3.65 & 2.54 & 3.15 & 1.54 & 2.50 & 3.17 & 1.88 \\
\hline
\end{tabular}

transport of these cations, accompanying wet southerly air masses (Camarero \& Catalan 1993; 1996), has more impact on bulk deposition than dust of local origin.

\subsection{Snow vs rain precipitation}

Various practical reasons make it more difficult to sample snow deposition than rainwater (e.g., Sevruk 1993); however, the deposition of snow and the substance load accumulated within the snow cover are very important at the MOLAR sites. There is a good deal of uncertainty as to the estimation of precipitation amounts, especially at high wind speed, and much effort has been made to optimise a sampling procedure (Lovblad 1996).

Two different sampling techniques used to evaluate the chemical load of deposited snow were compared in Austria, in the watershed of Gossenköllesee.

The accumulation period, i.e. the time span until the onset of snow melt, lasted from mid November 1997 to the beginning of April 1998. Snow cores were sampled at two sites in the catchment of Gossenköllesee. The water equivalent and the nitrate, ammonium and sulphate loads of the snow pack were compared with the respective cumulative amounts of weekly sampled precipitation (wet only).

Precipitation amounts collected with the wet-only sampler reached only 40 to $60 \%$ of the corresponding water equivalent values of the snow cover. This deficit was, however, offset by higher weighted mean sulphate and ammonium concentrations in the precipitation, resulting in ionic loads that compared fairly well with the respective loads in the snow pack (Tab. 11). The wetonly nitrate loads, in contrast, remained well below the snow pack values due to similar mean nitrate concentrations both in precipitation and in the snow cover (Fig. 7).
Apart from the methods used, a further point concerns the relative importance, in terms of quantity and quality, of snow and rain in the MOLAR site. A specific study was performed at Robiei, in the southern Swiss Alps, at 1890 m a.s.l. (Barbieri \& Mosello 1999). Sampling was performed on a weekly basis from July 1996 to July 1997; the total amount of precipitation was 1999 $\mathrm{mm}$, with the snow contribution about $49 \%$.

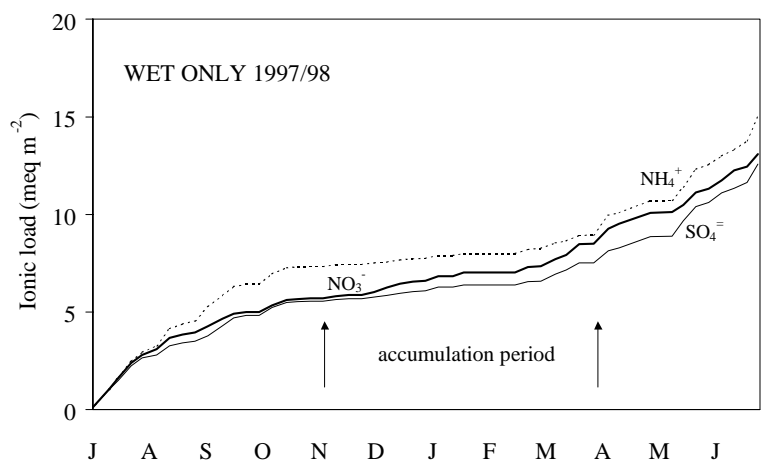

Fig. 7. Cumulative lines of nitrate, ammonium and sulphate loads in atmospheric deposition (wet only) at Gossenköllesee, July 1997 to June 1998. Arrows mark the snow accumulation period.

The comparison of the chemical composition of rain and snow (Fig. 8) shows a solute content lower in snow than in rain (41 and $\left.126 \mu \mathrm{eq} \mathrm{l}^{-1}\right)$; the difference is more marked for the ions of marine origin, which are below the quantification limit during the winter period. These differences are due to several factors.

The first is the varying stratification of the atmosphere in the course of a year. During the winter period the transport of ions from the lowlands and valleys, where the main sources of pollution (and the sea) are lo- 
cated, is very low due to the generally high stability of the atmosphere limiting the vertical mixing of air. During the summer period, in contrast, the contribution of ions transported from the lowlands is high due to enhanced vertical mixing of the atmosphere, determining concentrations of sulphate and ammonium 5 times higher than in winter, and two times higher for nitrate. A second factor responsible for the different composition of rain and snow is the greater light intensity in summer than in winter, increasing the oxidation of sulphur dioxide to sulphur trioxide (Calvert et al. 1985). A further point is the different scavenging by rain and snow of the sulphate present in the atmosphere (Mosello et al. 1988). In the specific case of Robiei the amount of deposition of the different ions reflects the differences in chemical composition, as the amounts of snow and rain precipitation are practically the same (Fig. 8).

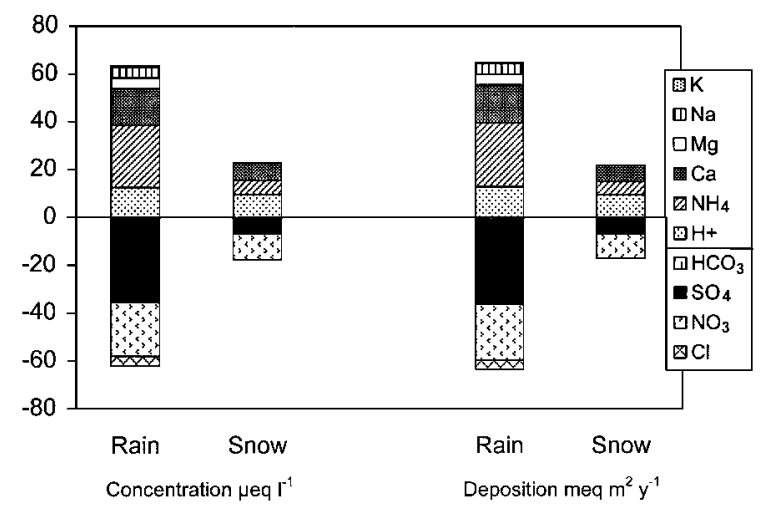

Fig. 8. Chemistry and amount of deposition of rain and snow in the site of Robiei/Laghetto Inferiore $(\mathrm{CH})$.

Similar seasonal variations in the chemistry of deposition were measured in other Alpine sampling stations (Graniga, Jörisee, Ritten): winter and early spring concentrations of sulphate, ammonium and nitrate are far lower than during summer (Puxbaum et al. 1991).

\subsection{Variation of lake water chemistry with depth and season}

The work package of the MOLAR project considered here included performing measurements on surface water. To understand how representative of the mean situation they are, it is important to compare these concentrations with those of deeper water. Surface water monitoring is less expensive and technically easier than sampling over the whole column, in particular in those areas without road access for transporting a boat or other equipment. As an index of the ion content of the water we chose conductivity, which is fairly well related to ion composition, at the end of the summer period, when water stratification, if any, is more likely to be found. The conductivity profiles measured in a group of MOLAR lakes are presented in figure 9.

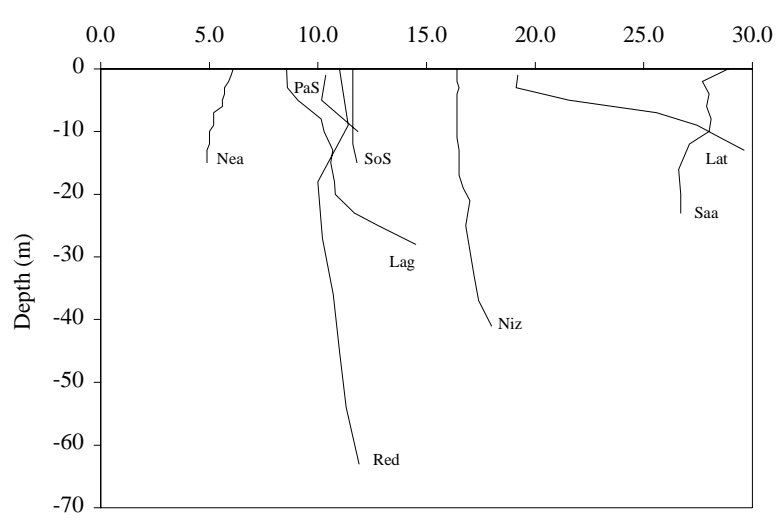

Fig. 9. Variation of conductivity $\left(\mu \mathrm{S} \mathrm{cm}^{-1}\right.$ at $\left.25^{\circ} \mathrm{C}\right)$ with depth in selected MOLAR lakes.

They show how surface values could be considered representative of the mean concentrations. In some cases (e.g., Laghetto Inferiore, Lago di Latte) conductivity values increase with depth, while in other lakes, like Øvre Neådalsvatn and Saanajärvi, it is the opposite. However, it must be pointed out that, particularly in the deepest lakes (depth $>20-30 \mathrm{~m}$ ), even a slight increase in solute concentration can affect the possibility of water mixing. These processes and their biological implications are considered in another WP of the MOLAR project (Patrick et al. 1998).

The most important event affecting water chemistry is the snow melt, which in all the lakes causes a more or less sharp decrease in alkalinity, $\mathrm{pH}$, calcium and major ion concentrations. Figures 10 and 11 show these variations in the Norwegian Lake Øvre Neådalsvatn, the Alpine lakes Paione Superiore and Gossenköllesee, and Redó, in the Pyrenees. The first three lakes clearly show the effects of the snowmelt, while in the case of Redó there are no variations, although they have been observed in years with a higher sampling frequency (Catalan 1992).

\subsection{Long-term variations in lake chemistry}

Although not included in the MOLAR project, the trend of chemical concentrations can be analysed by pooling the data of four lakes for which long-term series of data are available, thanks to continuity with the EU programs AL:PE1 and AL:PE2, as well as with national studies. In the case of Stavsvatn and Paione Superiore (Fig. 12), pH and alkalinity increased during 1986-1997, while the $\mathrm{SO}_{4}=$ concentration decreased. The trends were similar in Redó, except that pH slightly decreased in contrast with the increasing alkalinity. The Austrian lake Schwarzsee ob Sölden has had the same increase in $\mathrm{pH}$ and alkalinity since 1992, but, unlike the other lakes, it also shows a marked increase in sulphate concentration. 
Øvre Neådalsvatn
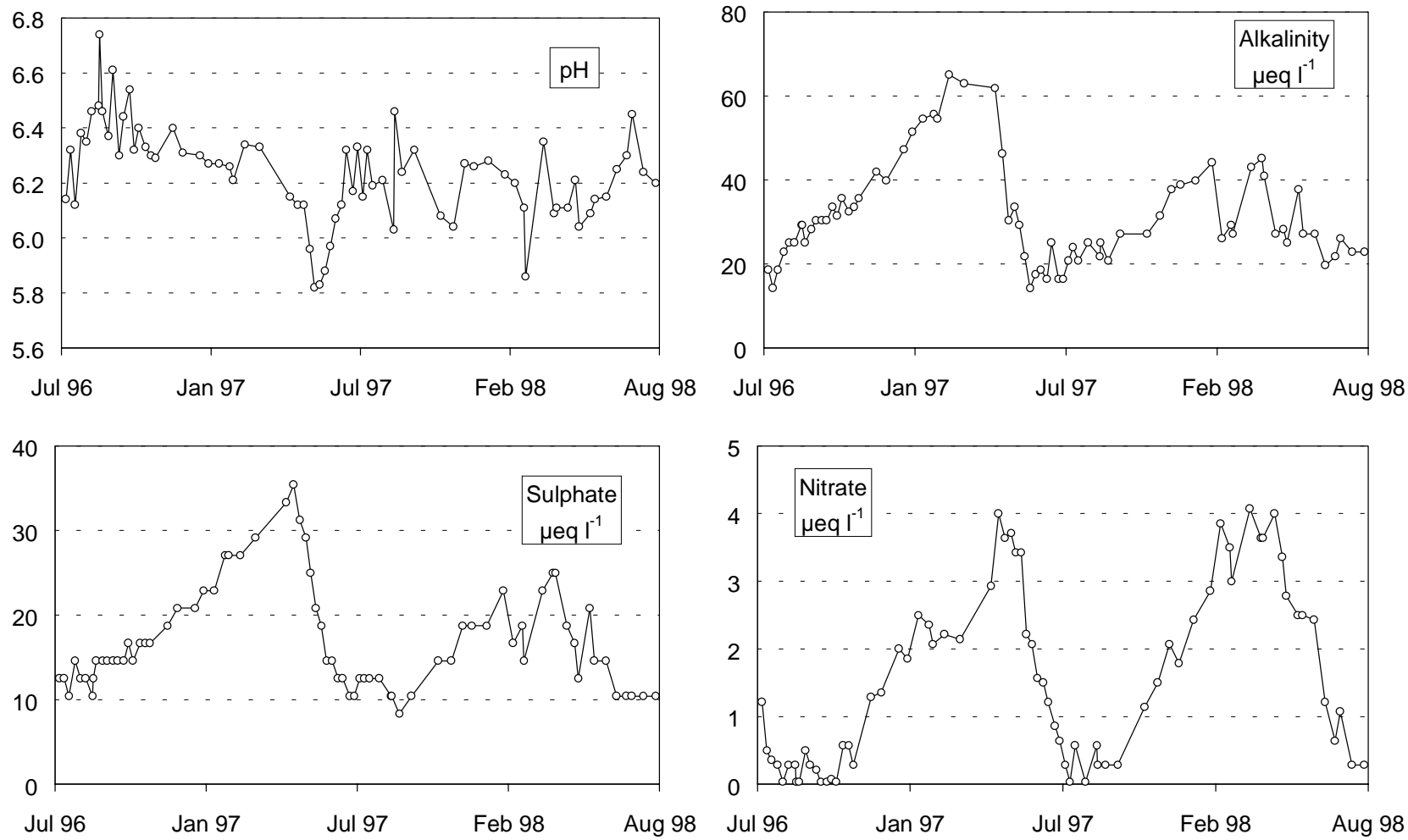

Lago Paione Superiore
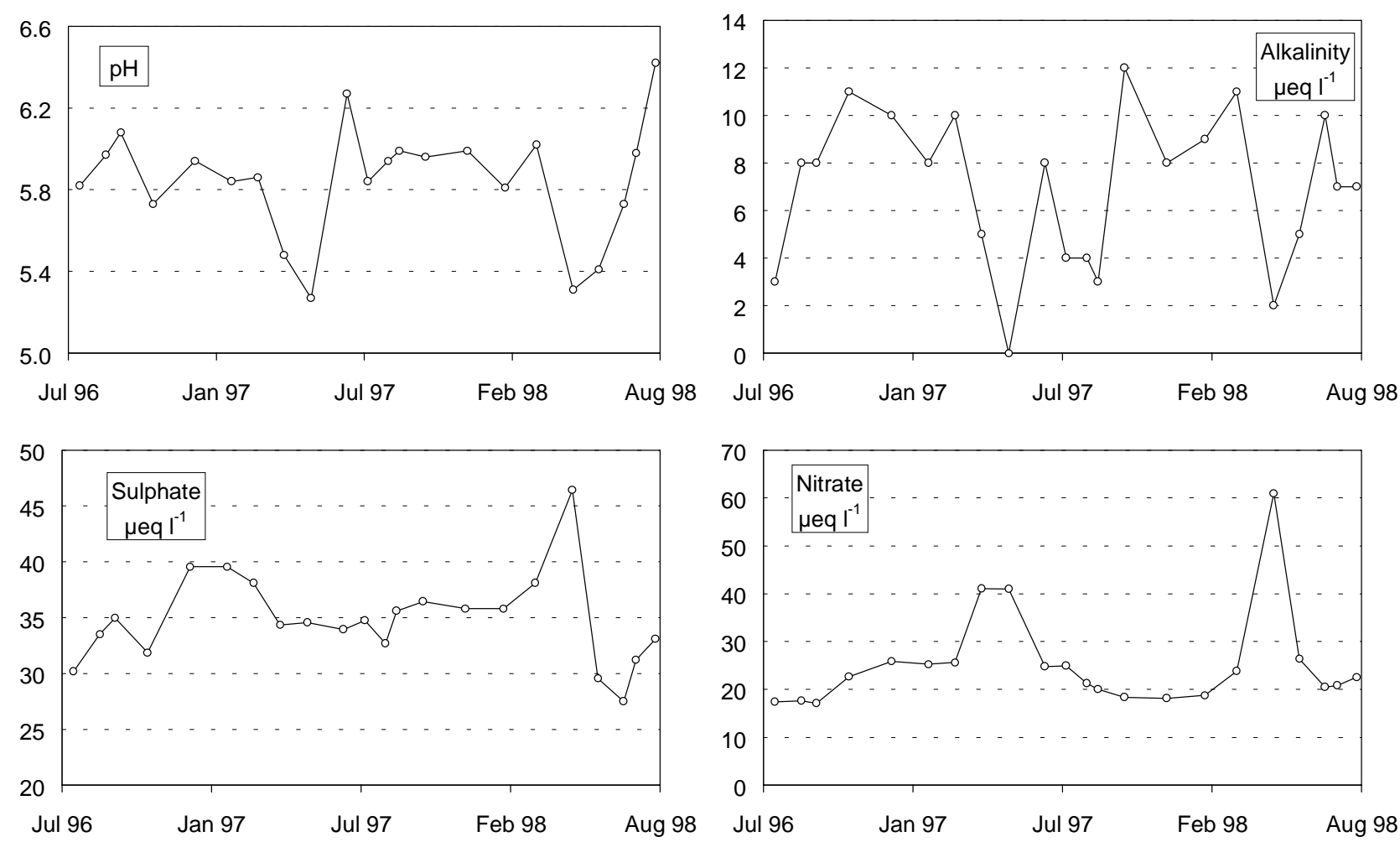

Fig. 10. Seasonal variations of $\mathrm{pH}$, alkalinity, sulphate and nitrate in lakes Øvre Neådalsvatn and Paione Superiore. 


\section{Gossenköllesee}
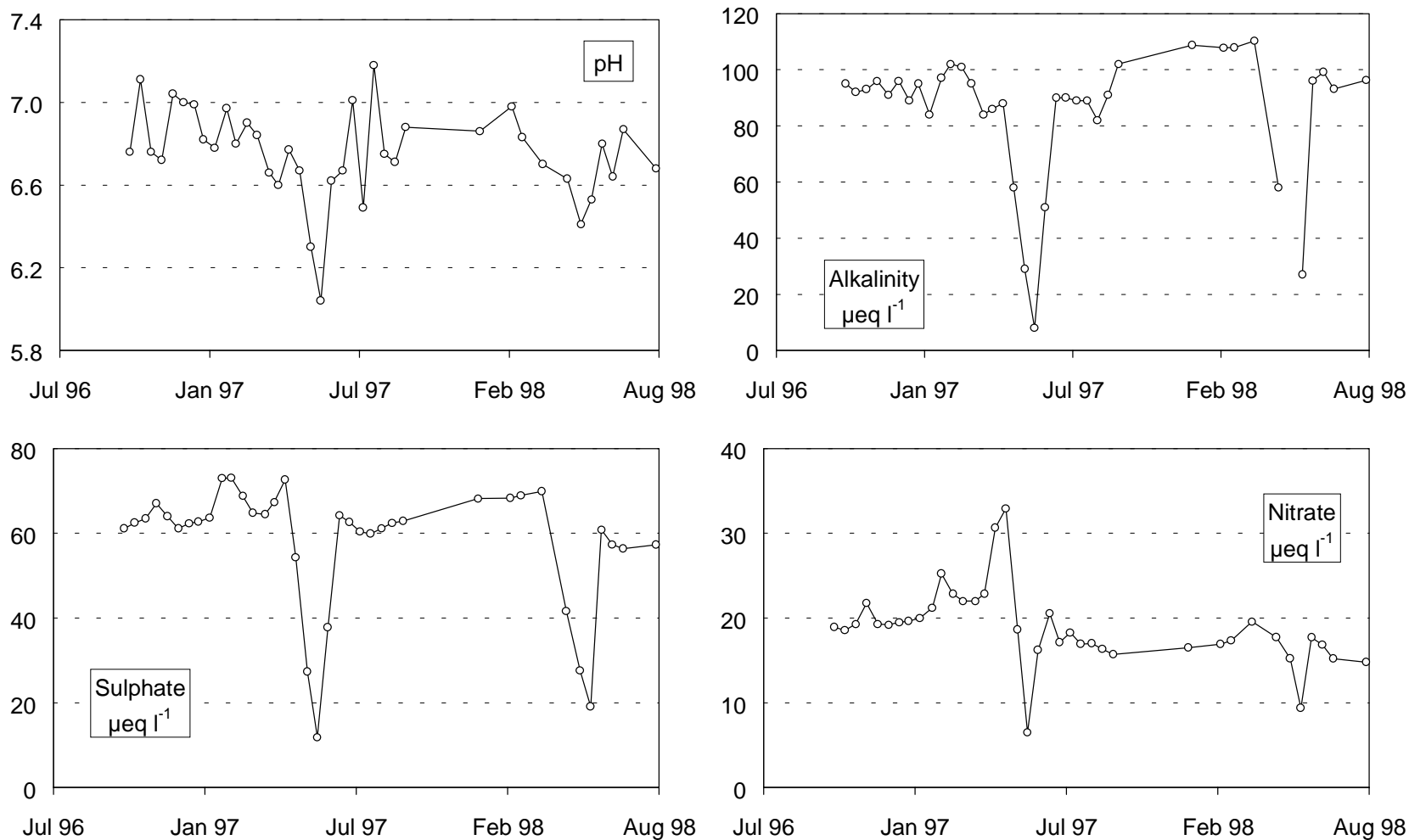

\section{Redó}
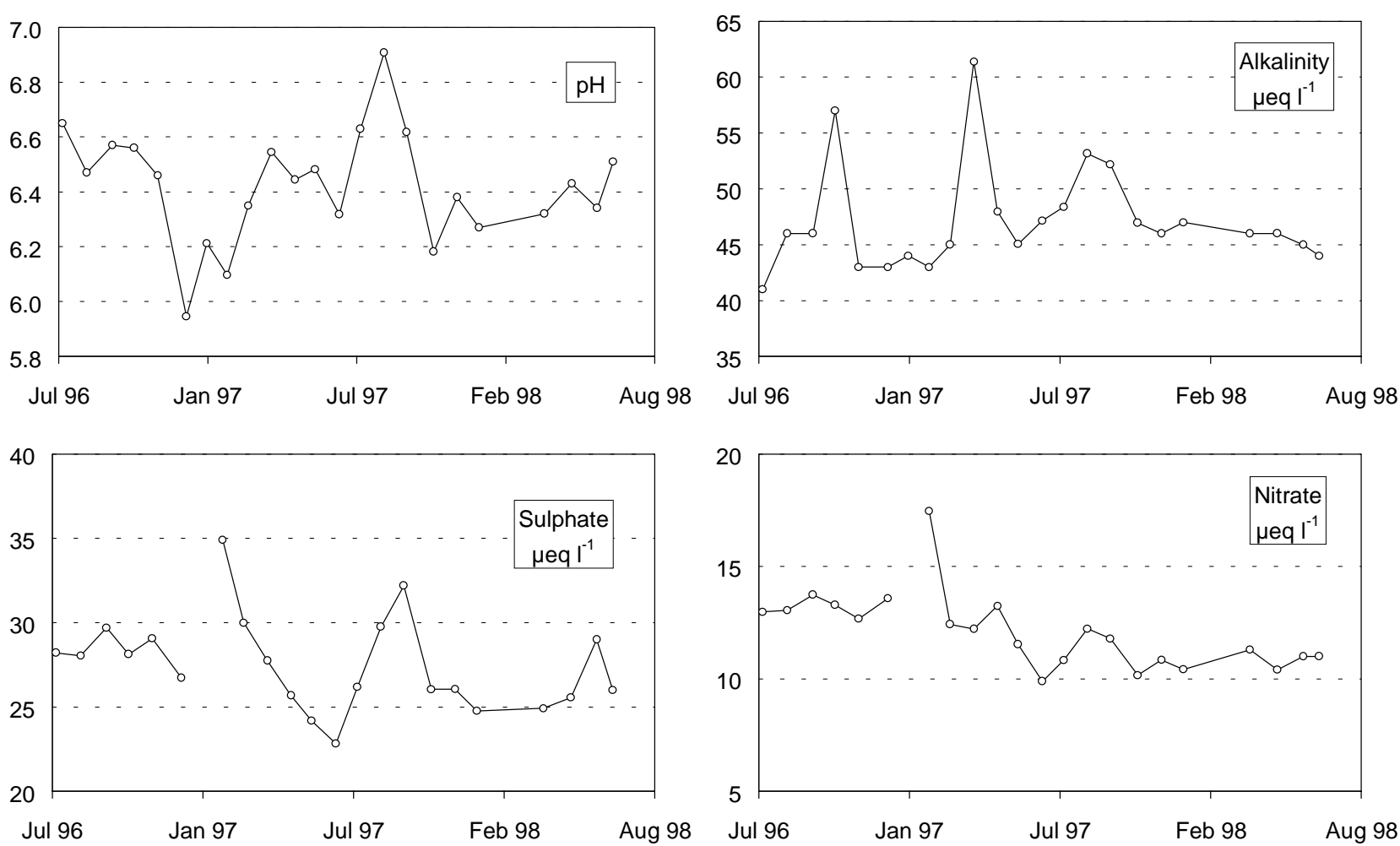

Fig. 11. Seasonal variations of $\mathrm{pH}$, alkalinity, sulphate and nitrate in lakes Gossenköllesee and Redó. 

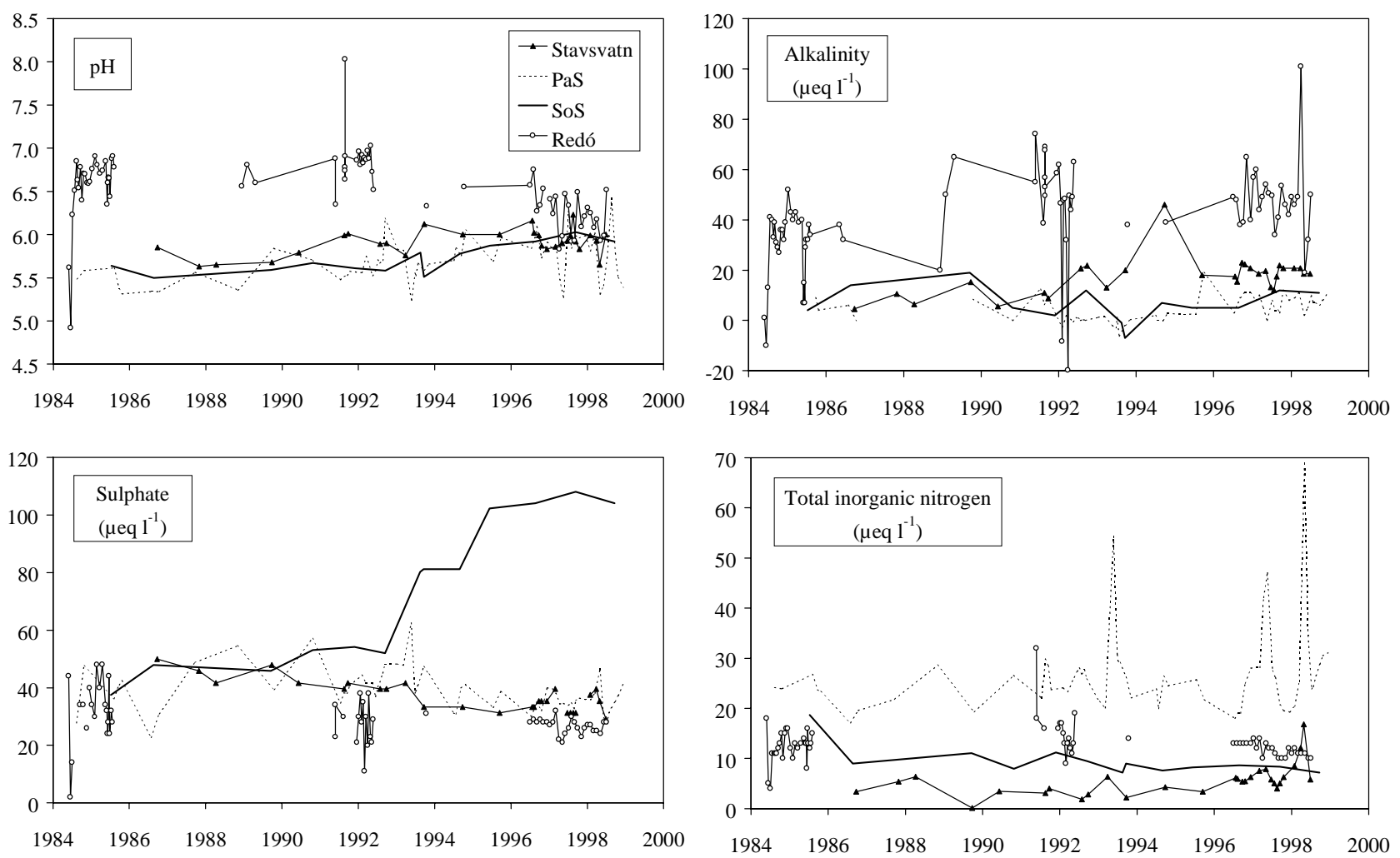

Fig. 12. Trend of $\mathrm{pH}$, alkalinity, sulphate and total inorganic nitrogen in lakes Stavsvatn, Paione Superiore (PaS), Schwarzsee ob Sölden (SoS) and Redó.

Concentrations of inorganic nitrogen show strong fluctuations in Paione Superiore, it was stable or had slightly decreased in Schwarzsee ob Sölden and Redó, and showed a slight increase during the last few years only in the case of Stavsvatn. Decline of nitrogen was recorded in the high altitude lakes in the Tatra Mountains during the last decade due to significant reduction of N emission in Central Europe (Kopáček et al. 1998).

For the Norwegian and Italian lakes, the trends of $\mathrm{pH}$, alkalinity and sulphate are related to a decrease in acidity and the $\mathrm{SO}_{4}{ }^{2}$ content of atmospheric deposition, whereas nitrogen is constant or in slight increase (Mosello et al. 1999; Henriksen et al. 1990). In the case of the Austrian lake the increase in sulphate seems to be related to higher temperatures during summer, producing a higher weathering rate (Sommaruga-Wograth et al. 1997; Koinig et al. 1998). The Spanish lake showed, on the one hand, an apparent increase in the weathering rate (with an increase of calcium strongly related to alkalinity) and, on the other hand, a decrease in atmospheric sulphate deposition.

Other long-term data are available for some of the MOLAR lakes (e.g., Lochnagar, Długi Staw); an overall analysis of these trends and what produced them is the topic of a further EU project.

\section{CONCLUSIONS}

In Europe, remote mountain areas have considerable importance both as regards the percentage of the surface area they occupy and in terms of their value as a natural and recreative resource. In such a densely populated continent, and one in which, more than in others, natural ecosystems have been altered as a consequence of human impact, these areas are today particularly important. They also have great hydrological importance, as the rivers which feed the lakes, also the lakes of the plains, rise here, providing a reserve of high quality water for a number of civil and industrial uses.

These areas are for the most part far from sources of pollution, though phenomena of atmospheric transport of pollutants mean that they are not inevitably immune from the deposition of these substances. Studying the quality of the water in remote areas can therefore yield important information on levels of background pollution, to the extent that these lakes can be used as early warning systems for the state of health of the environment.

The results of this part of the MOLAR project highlight some of these aspects. The chemistry of atmospheric deposition collected in sites which are represen- 
tative of remote areas in the whole of Europe, from the extreme north of Scandinavia to the southern Alps and the Pyrenees, shows that the solutes present may be marine $\left(\mathrm{Na}^{+}, \mathrm{Cl}^{-}\right.$, fraction of $\mathrm{Mg}^{++}$and $\left.\mathrm{SO}_{4}{ }^{+}\right)$, terrigenous $\left(\mathrm{Ca}^{++}, \mathrm{K}^{+}\right.$, fraction of $\mathrm{Mg}^{++}, \mathrm{HCO}_{3}^{-}$when present $)$, and deriving from fuel-burning emissions and intensive stock-breeding $\left(\mathrm{SO}_{4}{ }^{2}, \mathrm{NH}_{4}{ }^{+}, \mathrm{NO}_{3}{ }^{-}\right)$. The acidity of the depositions depends on the different importance in percentage terms of each component, especially the terrigenous one and that deriving from anthropogenic emissions. The results show marked variability in the different areas of Europe, with the worst situation in central Europe and in some Alpine stations. But even in areas like Scandinavia, for example, a gradient is clear from the southernmost station, Møsvatn, to Kårvatn, situated in the central part of Norway, which is less affected by long-range transported air pollution. Seasonal variations emerged as having great importance, both in the chemical composition and in the volume of water, and it must be borne in mind that, during winter, most of the deposition is in form of snow, so all the ionic load, including pollutants, is released in a very short space of time, during snowmelt, causing marked pulses in the chemistry of rivers and lakes. The relative importance of episodic events as opposed to phenomena which develop continuously in the ecosystem, is a major question requiring particular study methods.

The chemistry of these lakes is largely determined by three factors: (1) atmospheric loads, (2) weathering processes, and (3) interaction with the vegetation in the watershed, scarce but not negligible, and with the biology of the lake waters. The geological characteristics of many watersheds, made up largely of poorly soluble rocks, means that many of the considered lakes are sensitive to acidification, with some of them showing clear signs of alteration. The interaction between the vegetation cover of the watershed and the chemistry of the water is particularly important when determining nitrogen levels. In general, atmospheric nitrogen loads are high, so that nitrate is present in the water of many remote lakes in relatively high concentrations. This is particularly true with regard to the lakes in the Tatra Mountains, but also for those in the Alps. Gradient on nitrate in the Tatra Mountains lakes is the result of the amount of vegetation in the watershed and the acidification status of each lake (Kopáček et al. 1995). It is also striking how ammonium, which is present in significant quantities in atmospheric loads, disappears almost completely in surface waters. It is known that the uptake of ammonium from vegetation is a further source of acidity, and that the oxidation of ammonium to nitrate produces acidity. For this reason, $\mathrm{pH}$ is not the best indicator of the real acidifying capacity of atmospheric deposition; more detailed studies are, however, required. A comparison of the atmospheric load of acidity (real and potential) and of nitrogen with the respective critical values will be included in the MOLAR project.
The long-term evolution of water chemistry, although considered only for four lakes, clearly shows that the situation is far from the steady state. On one hand, the increase in alkalinity and $\mathrm{pH}$ values is a positive indication of the success achieved in reducing the load of acidity from atmospheric deposition, the result of the concerted efforts of most of the European countries. On the other hand, the considerable variations in nitrate and the different levels found in the lakes are evidence that the problems persist, and that it is essential that studies on and monitoring of such sensitive systems should continue. A further issue which has not been entirely clarified is that of the mechanisms responsible for the increase of alkalinity in the lakes. Is the decrease of acid deposition from the atmosphere a sufficient cause, or do climatic variations, as suggested by some results (Sommaruga-Wögrath et al. 1997) also play a part?

These questions, and the close relationships existing between water chemistry and biology, emphasise how the presentation of the chemical data in this paper is merely a stage in a more general process of elaboration and evaluation of the data collected in the framework of the MOLAR project. This special volume of the Journal of Limnology devoted to trophic relations shows how complex and in continual evolution is the picture emerging from our data.

Last but not least, the three years of collaboration within the MOLAR project, added to the previous collaboration in the AL:PE 1 and AL:PE 2 projects, has resulted in the establishment of excellent scientific and personal relationships among experts in different disciplines and in different countries, both inside and outside the European Union. We believe that this is at least as important as the scientific results which have been produced, since the formation of relationships of mutual respect between people form a basis for important future scientific collaboration, which will be an essential prerequisite for tackling the environmental problems of the next few years.

\section{REFERENCES}

A.P.H.A., A.W.W.A., W.P.C.F. 1992. Standard Methods for the examination of water and wastewater. Am. Publ. Healt ass., Washington.

Barbieri, A. \& R. Mosello. (1999). Recent evolution in chemistry and mass budget of a high altitude lake in southern Alps (Laghetto Inferiore, Switzerland). $J$. Limnol:: submitted.

Battarbee, R.W., B.M. Wathne, M. Joannessen, R. Mosello, S. Patrick, G.G. Raddum, B.O. Rosseland, J.O. Grimalt, J. Catalan, R. Hofer, R. Psenner, R. Schmidt, A. Lami, N.G. Cameron, N.L. Rose, V.J. Jones \& H.J.B. Birks. 1997. Remote mountain lakes as indicators of environmental change. Proceedings of SETAC Conference, Copenhagen, June 1995: in press.

Calvert, J.G., A.L. Lazrus, G.L. Kok, B.G. Heikes, J.G. Walega, J. Lind \& C.A. Cantrell. 1985. Chemical mechanism of acid generation in the troposphere. Nature, 317 (6032): 27-35. 
Camarero, L. \& J. Catalan. 1993. Chemistry of bulk precipitation in the Central and Eastern Pyrenees (Northeast Spain). Atmos. Environ., 27A: 83-94

Camarero, L. \& J. Catalan. 1996. Variability in the chemistry of precipitation in the Pyrenees (northeastern Spain): Dominance of storm origin and lack of altitude influence. J. Geophys. Res., 101: 29491-29498.

Carratala, A., J. Bellot, A. Gomez \& M. Millan. 1996. African dust influence on rainwater on the Easten coast of Spain. In: Guerzoni, S. \& R. Chester (Eds), The impact of desert dust across the Mediterranean. Kluwer, Dordrech: 323332 .

Catalan, J. 1992. Evolution of dissolved and particulate matter during the ice-covered period in a deep, high mountain lake. Can. J. Fish. aquat. Sci., 49: 945-955.

Draaijers, G.P.J. \& J.W. Erisman. 1993. Atmospheric sulphur deposition to forest stands: throughfall estimates compared to estimates from inference. Atmosph. Environ., 27A: 4355.

Draaijers, G.P.J., J.W. Erisman, G. Lövblad, T. Spranger \& E. Vel. 1998. Quality and uncertainty aspects of forest deposition estimation using througfall, stemflow and precipitation measurements. TNO-report - R98/093, Apeldoorn, NL: 73 pp.

Fresenius W., K.E. Quentin \& W. Schneider (Eds). 1988. Water Analysis. Springer-Verlag Berlin: 804 pp.

Guerzoni, S. \& R. Chester (Eds). 1996. The impact of desert dust across the Mediterranean. Kluwer, Dordrecht: 389 pp.

Henriksen, A., E. Joranger \& A. Semb. 1990. The contribution of nitrogen to acidification. The National Environmental Monitoring Programme Report 408/90, State Pollution Control Authority, Oslo.

Hovind, H. 1997. Intercomparison 9711. pH, K25, alkalinity, nitrate+nitrite, chloride, sulphate, calcium, magnesium, sodium, potassium, total aluminium, aluminium-reactive and nonlabile, TOC and COD-Mn. NIVA Report, ISBN 82-577-3284-2, Oslo: 57 pp.

Koinig, K.A., R. Schmidt, S. Sommaruga-Wögrath, R. Tessadri \& R. Psenner. 1998. Climate change as the primary cause for $\mathrm{pH}$ shifts in a high alpine lake. Water Soil Air Pollut., 167-180.

Kopáček, J., L. Procházková, E. Stuchlík \& P. Blažka. 1995. The nitrogen-phosporus relationship in mountain lakes: influence of atmospheric input, watershed and $\mathrm{pH}$. Limnol. Oceanogr., 40: 930-937.

Kopáček, J., J. Hejzlar, E. Stuchlík, J. Fott \& J. Veselý. 1998. Reversibility of acidification of mountain lakes after reduction in nitrogen and sulphur emissions in Central Europe. Limnol. Oceanogr., 43: 357-361.

ISO 10566: 1994. Water quality - Determination of aluminium - Spectrometric method using pyrocathecol violet.

Lovblad, G. 1996. Report from the Meeting of the Expert Panel on Deposition in Stenungsund. Sweden, 21-22 October 1996.

Marchetto, A., M. Bianchi, H. Geiss, H. Muntau, G. Serrini, G. Serrini Lanza, G.A. Tartari \& R. Mosello. 1997. Performances of analytical methods for freshwater analysis assessed through intercomparison exercises. I. Total alkalinity. Mem. Ist. ital. Idrobiol., 56: 1-13.

Mosello, R. \& B.M. Wathne. 1997. Surface water. Chemical analysis of major ions and nutrients. Analytical quality control. In: Wathne, B.M (Ed.). MOLAR. Measuring and modelling the dynamic response of remote mountain lake ecosystem to environmental change: A programme of Mountain lake Research. MOLAR Project Manual. NIVA Report 0-96061: 24-45.

Mosello, R., A. Marchetto \& G.A. Tartari. 1988. Bulk and wet atmospheric deposition chemistry at Pallanza (N. Italy). Water Soil Air Pollut., 42: 137-151.
Mosello, R., B.M. Wathne \& S. Van der Berg. 1997b. Surface water. Sampling and analysis protocol. In: Wathne, B.M (Ed.), MOLAR. Measuring and modelling the dynamic response of remote mountain lake ecosystem to environmental change: A programme of Mountain lake Research. MOLAR Project Manual. NIVA Report 0-96061: 20-23.

Mosello, R., T. Berg, U. Nickus \& P. Appleby. 1997a. Atmospheric deposition. Sampling and analysis of direct deposition, snow pack and soil cores. In: Wathne, B.M (Ed.). MOLAR. Measuring and modelling the dynamic response of remote mountain lake ecosystem to environmental change: A programme of Mountain lake Research. MOLAR Project Manual. NIVA Report 0-96061: 7-18.

Mosello, R., A. Marchetto, M.C. Brizzio, G.A. Tartari \& M. Rogora. 1999. Pluriannual evolution of the hydrochemistry of two alpine lakes (Lake Paione Inferiore and Lake Paione Superiore, Ossola Valley) in relation to atmospheric loads. J. Limnol., 58(1): 43-49.

Mosello, R., M. Bianchi, H. Geiss, A. Marchetto, G. Serrini, G. Serrini Lanza, G.A. Tartari \& H. Muntau. 1998b. AQUACON-MedBas Subproject $N^{\circ} 5$. Freshwater analysis. Intercomparison 1/97. Joint Res. Centre European Commission. Rep. EUR 18075 EN: 66 pp.

Mosello, R., M. Bianchi, M.C. Brizzio, H. Geiss, W. Leyendecker, A. Marchetto, G. Serrini, G. Serrini Lanza, G.A. Tartari \& H. Muntau. 1998a. AQUACON-MedBas Subproject No. 6. Acid rain analysis. Intercomparison 1/97. Joint Res. Centre European Commission, Rep. EUR 18135 EN: 65 pp.

Nickus U., H. Thies, M. Kuhn \& R. Psenner. 1998. The snow cover at a headwater site in the Tyrolean Alps: seasonal and local variability of atmospheric trace substances in the snow pack. In: Tappeiner, U. et al. (Eds), Hydrology, Water Resources and Ecology of Mountain Areas. HeadWater '98 Conference, Meran/Merano, Italy, April 1998: 39-42.

Nickus, U., M. Kuhn, U. Baltensperger, R. Delmas, H. Gäggeler, A. Kasper, H. Kromp-Kolb, F. Maupetit, A. Novo, F. Pichlmayer, S. Preunkert, H. Puxbaum, G. Rossi, W. Schöner, M. Schwikowski, P. Seibert, M. Staudinger, V. Trockner, D. Wagenbach \& W. Winiwarter. 1997. SNOSP: Ion deposition and concentration in high alpine snow packs. Tellus, 49B: 56-71.

Patrick, S., R.W. Battarbee, B. Wathne \& R. Psenner. 1998. Measuring and modelling the dynamic response of remote mountain lake ecosystems to environmental change: an introduction to MOLAR project. Proc. of the HeadWater '98 Conference, Meran/Merano, Italy, April 1998, IAHS Publ. 248: 403-410.

Puxbaum H., A. Kovar \& M. Kalina. 1991. Chemical composition and fluxes of wet deposition at elevated sites (700$3105 \mathrm{~m}$ a.s.1.) in the easterly Alps (Austria). NATO ASI Series, G28: 273-297.

Rogeberg, E.J.S \& A. Henriksen. 1985. An automatic method for fractionation and determination of aluminium species in freshwater. Vatten, 41: 48-53.

Sevruck, B. 1993. Checking precipitation gauge performance. In: Couling, S. (Ed.), Measurement of airborne pollutants. Butterworth Heinemann, Oxford (UK): 89-107.

Sommaruga-Wögrath, S., K.A. Koinig, R. Schmidt, R. Sommaruga, R. Tessadri \& R. Psenner. 1997. Temperature effects on the acidity of remote alpine lakes. Nature, 387: 64-67.

Van Leeuwen, N.P.M., G.P.J. Draaijers \& J.W. Erisman. 1996. Mapping wet deposition of acidifying components and base cations over Europe using measurements. Atmosph. Environm., 30: 2495-2511.

Wathne, B.M. \& H.E. Hansen. 1997. MOLAR. Measuring and modelling the dynamic response of remote mountain lake ecosystem to environmental change: A program of Moun- 
tain lake Research. MOLAR Project Manual. NIVA Report 0-96061, Oslo.

Wathne, B.M., S.T. Patrick \& N. Cameron. 1997. AL:PE Acidification of mountain lakes: palaeolimnology and ecology. Part 2 - Remote mountain lakes as indicators of air pollution and climate change. NIVA Report 3638-97, Oslo.
Wathne, B.M., S.T. Patrick, D. Monteith, \& H. Barth. 1995. AL:PE - Acidification of mountain lakes: palaeolimnology and ecology. AL:PE 1 report for the period April 1991April 1993. European Commission, Ecosystem Research Report 9, Luxembourg. 